TITLE:

\title{
Modeling and Control of a Snake- Like Robot Using the Screw-Drive Mechanism
}

\section{$\operatorname{AUTHOR}(\mathrm{S})$ :}

Fukushima, Hiroaki; Satomura, Shogo; Kawai, Toru; Tanaka, Motoyasu; Kamegawa, Tetsushi; Matsuno, Fumitoshi

\section{CITATION:}

Fukushima, Hiroaki ... [et al]. Modeling and Control of a Snake-Like Robot Using the ScrewDrive Mechanism. IEEE Transactions on Robotics 2012, 28(3): 541-554

\section{ISSUE DATE:}

2012-06

URL:

http://hdl.handle.net/2433/158351

\section{RIGHT:}

(c) 2012 IEEE. Personal use of this material is permitted. Permission from IEEE must be obtained for all other uses, in any current or future media, including reprinting/republishing this material for advertising or promotional purposes,

creating new collective works, for resale or redistribution to servers or lists, or reuse of any copyrighted component of this work in other works.; This is not the published version. Please cite only the published version.; この論文は出版社版

でありません。引用の際には出版社版をご確認ご利用ください。 


\title{
Modeling and Control of a Snake-like Robot Using the Screw Drive Mechanism
}

\author{
Hiroaki Fukushima, Member, IEEE, Shogo Satomura, Toru Kawai, Motoyasu Tanaka, Tetsushi Kamegawa, \\ and Fumitoshi Matsuno, Member, IEEE
}

\begin{abstract}
In this paper, we develop a new type of snake-like robot using screw-drive units connected by active joints. The screw drive units enable the robot to generate propulsion on any side of the body in contact with environments. Another feature of this robot is the omni-directional mobility by combinations of screws' angular velocities. We also derive a kinematic model and apply it to trajectory tracking control. Furthermore, we design a front-unit-following controller, which is suitable for manual operations. In this control system, operators are required to command only one unit in the front, then commands for the rest of the units are automatically calculated to track the path of the preceding units. Asymptotic convergence of the tracking error of the front-unit-following controller is analyzed based on a Lyapunov approach for the case of constant curvature. The effectiveness of the control method is demonstrated by numerical examples and experiments.
\end{abstract}

Index Terms-snake-like robot, screw drive mechanism, path tracking, search and rescue

\section{INTRODUCTION}

M OBILE robots for search and rescue operations in hazardous environments have been actively studied in recent years. One promising type of rescue robots is the so called snake-like robot, which is typically composed of three or more segments connected serially. Because of the long and slender shape, snake-like robots are expected to be effective for searches in narrow spaces and over rubbles in quakedevastated regions, etc [1], [2]. Also, snake-like robots for pipe inspection have been reported in the literature [3], [4]. A conventional way of locomotion for snake-like robots is the one by undulations, which imitates real snakes' movements [5]-[15]. However, this type of locomotion needs a width for undulations, which is larger than the width of the robot.

On the other hand, snake-like robots driven by crawler mechanisms have been developed [1], [2]. One limitation of typical crawler-type robots arises in vertically narrow spaces, where the upper part of the robots could hit the ceiling. In those cases, the robots could be stuck easily, since the upper and lower parts of the crawlers drive the robot in opposite

This paper was presented in part at the IEEE International Conference on Robotics and Automation, Rome, Italy, April 2007 and the IEEE/RSJ International Conference on Intelligent Robots and Systems, Nice, France, September 2008.

H. Fukushima and F. Matsuno are with the Department of Mechanical Engineering and Science, Kyoto University, Kyoto, Japan (e-mail: fuku@me.kyotou.ac.jp)

S. Satomura and M. Tanaka are with Canon Inc., Tokyo, Japan

T. Kawai is with Honda R\&D Co, Ltd, Saitama, Japan

T. Kamegawa is with the Department of Natural Science and Technology, Okayama University, Okayama, Japan directions. To overcome this limitation, recent studies have proposed snake-like robots having crawlers on both upper and lower sides of the body [16], [17].

Locomotion mechanisms related to the robot in this paper are found for pipe inspection robots [18], [19], [20]. While they move by rotating a screw-like device, they are composed of one or two units and have a quite different structure from most snake-like robots. On the other hand, snake-like robots for pipe inspection are also studied in the literature [3], [4]. They form a sinusoidal wave using the whole body and move forward by switching the units pushing the pipe wall. Since these robots are designed specifically for inspection of small diameter pipes, they are not necessarily suitable for other applications such as search and rescue operations.

In this paper, we develop a new type of snake-like robot using the screw drive mechanism. The original concept is reported in our patent [21]. This robot is composed of screw drive units, connected by active joints serially. Since propulsion is generated by rotating the screws, undulation is not necessary to move. Thus, this robot can go into spaces as narrow as the width of the body. Also, it is expected that this robot does not get stuck easily even if the upper part of the body hit the ceiling, since the upper part of the screw units drive the body in the same direction as the lower part. Furthermore, unlike most existing snake-like robots, it can move in any direction by a proper combination of screws' angular velocities.

As the first step towards the control system design of the robot potentially having such attractive properties, we derive a kinematic model in the case where the robot does not contact with the environment except for the ground. Due to the

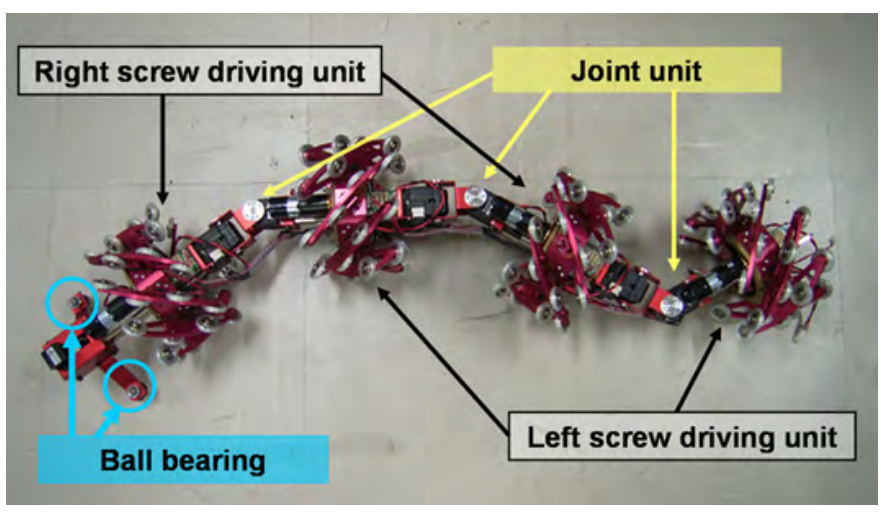

Fig. 1. Snake-like robot using the screw drive mechanism 
switching of the passive wheels in contact with the ground, the motion of the robot is complex even if the ground is flat and horizontal. In order to derive a simple kinematic model for control design, we represent the behavior of the screw unit using a velocity constraint at the center of the unit. While this velocity constraint is quite different from that of the conventional snake-like robots due to the screws, a kinematic model can be derived in the same way as the conventional snake-like robots moving by undulations [6]-[8], once the velocity constraint is obtained. To examine the validity of the model, both feedback and feedforward controllers designed using the model, are applied to the robot.

Even if the feedback controller to steer the robot to the target state is designed, a hard problem remained is how to determine the target state of the robot. For searches in narrow spaces, human operators typically need to determine the target state. However, it is hard for operators to give commands for all joints as well as the head position and orientation, such that the shape of the robot is fit to the narrow space.

In [1], a front-unit-following control system has been implemented to reduce difficulties in manual operations of a crawlertype snake robot. The operators are required to command only one unit in the head of the robot, then commands for the rest of the units are automatically calculated to track the path of the preceding units. While the effectiveness of the control law has been demonstrated by experiments, theoretical analysis on the tracking performance is still a challenging issue. Also, it is not straightforward to apply the method in [1] to the robot using the screw drive mechanism, due to the difference of the locomotion mechanism. Related to the frontunit-following control of snake robots, path-tracking control methods for articulated vehicles have been studied in the literature (see e.g. [22]-[24]). However, these methods assume that the target path for each unit is given, since they are based on feedback of tracking error from the target path. Thus, in order to apply these methods to front-unit-following control, the target path needs to be estimated based on the memory of the past commands to the front unit, which is difficult in many cases due to the computational burden.

In this paper, we design a front-unit-following control law using the only current velocity commands to the front unit. More precisely, the velocity of each unit is determined by assuming that the transition rate of curvature of the target path is sufficiently small in a local section between two consecutive joints of the robot, and that each unit is currently on the target path. Since this implies that a rapid change of curvature of the target path causes a large tracking error, it is important to find conditions where off-tracking can be recovered by the proposed control law. Thus, we also analyze the asymptotic convergence of the tracking error based on a Lyapunov approach for the case where the curvature of the target path is constant. The effectiveness of the control method is demonstrated by simulations and experiments including the cases where the curvature of the target path is not constant.

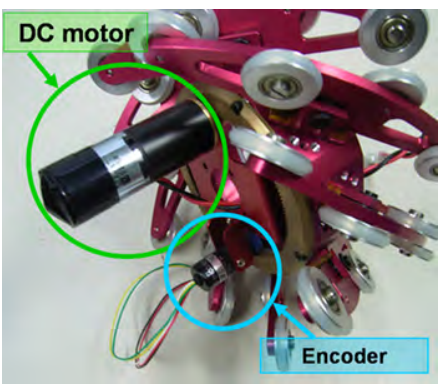

Fig. 2. Screw drive unit

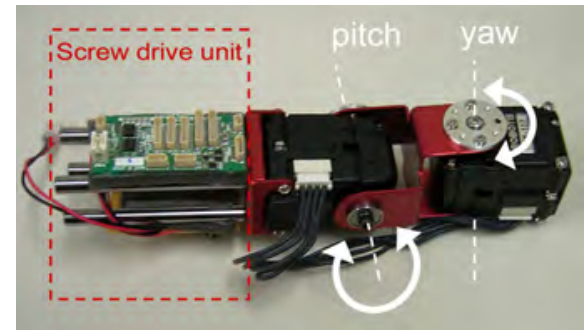

Fig. 3. Joint unit

\section{Snake-Like Robot with Screw Drive Mechanism}

\section{A. Outline of the robot system}

Fig. 1 shows a prototype of the snake-like robot using the screw drive mechanism. The robot is composed of two types of screw drive units, i.e. "left" and "right" screw units. In Fig. 1 , right and left units are connected alternatively from the head to the tail. The screw drive units are connected by 2-degreeof-freedom active joints. Moreover, a caster with ball bearings is set up at the head of the robot to prevent the body inside the unit from rotating instead of the screws, when the shape of the robot is straight. As shown in Fig. 2, each screw drive unit has a DC motor (A-max22, Maxon) inside to rotate the screw which is the outer part of the unit.

Fig. 3 shows a joint unit, which has two motors (Dynamixel DX-117, Robotis) for pitch and yaw angles. The range of movement of each motor is constrained to $\pm \frac{\pi}{2}$ [rad]. Feedback controllers for angular position and velocity are included inside the motors. All the motors for joint units are connected

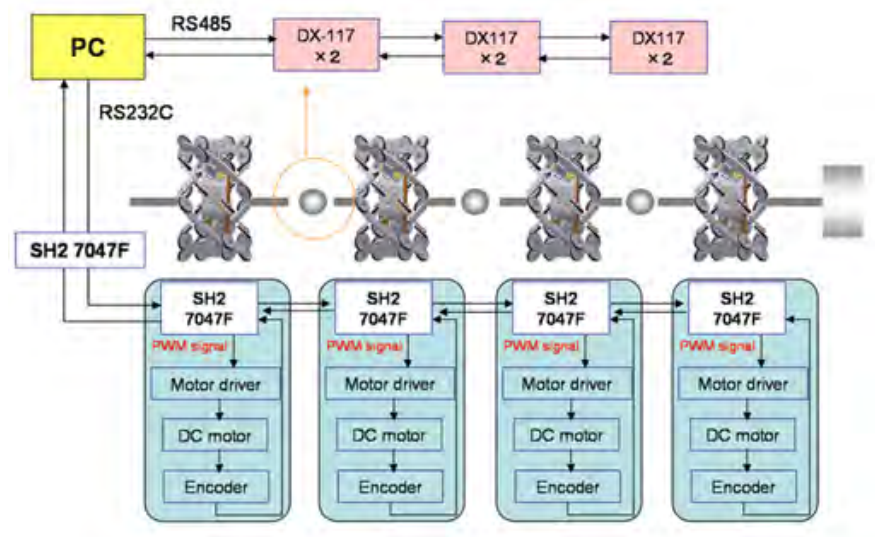

Fig. 4. Schematic of control system 

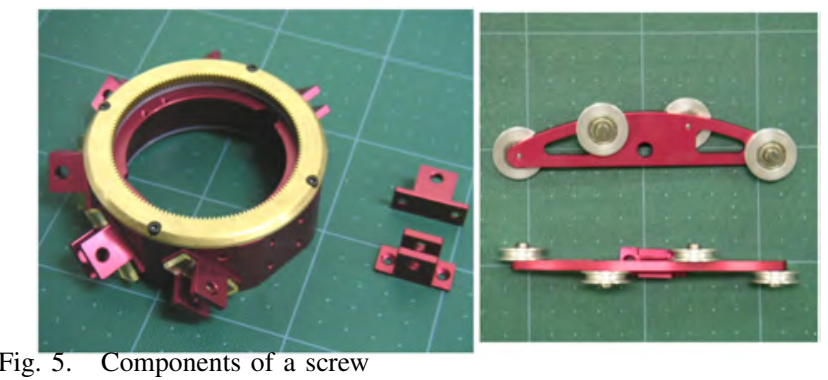

Fig. 5. Components of a screw
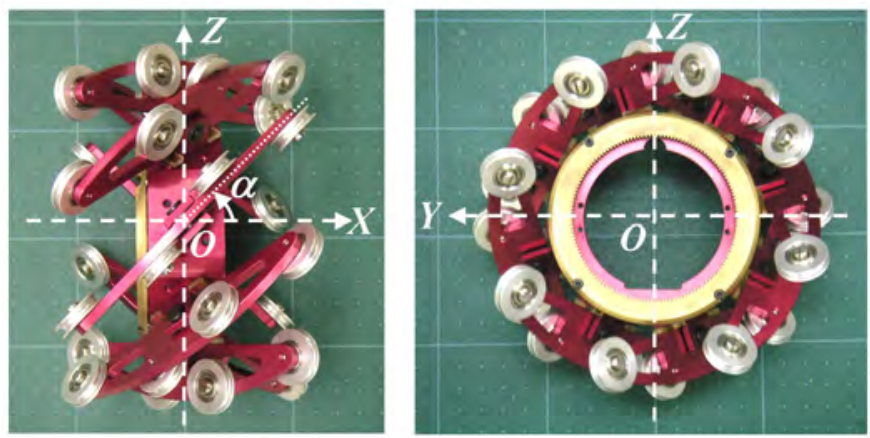

Fig. 6. Side and front views of a screw

in a daisy chain and communicate each other using RS485. Note that the pitch angle of each joint is controlled to 0 [rad], since we only consider the cases where the ground is flat in this paper.

Fig. 4 illustrates the structure of control system, which is divided into two main parts, the screw drive unit part and the joint unit part. The screw drive unit part communicates with a personal computer (PC) by using RS232C, and the joint unit part communicates with the PC by using RS485. For velocity control of a screw, a target value of the angular velocity is first sent from the $\mathrm{PC}$ to the microcomputer (TITech SH2 Tiny Controller, HiBot). Then, a pulse width modulation (PWM) signal is given from the microcomputer to the motor driver (1Axis DC Power Module, HiBot) to drive the DC motor. Count values of the encoders (MEH-9-360PC, Microtech Laboratory) are obtained by the microcomputers as rotation angle data of the screw part. One microcomputer is used for each screw drive unit, and another one attached to the tail of the robot is used for a relay between the units and the PC. The microcomputers communicate each other by using Controller Area Network (CAN).

\section{B. Screw drive unit}

A screw drive unit (Fig. 2) is composed of the screw part (Fig. 6) which actually rotates, and the inner body which is equipped with a DC motor to drive the screw part. The screw part is composed of a ring shaped part as shown on the left of Fig. 5, which is substantially a hollow regular octagonal prism having a ring gear in front. A blade as shown on the right of Fig. 5 is attached at the center of each side of the octagonal prism. Four passive wheels are attached to each blade. Note that each passive wheel has a rubber ring around the rim for providing more friction, as shown in Fig. 2.

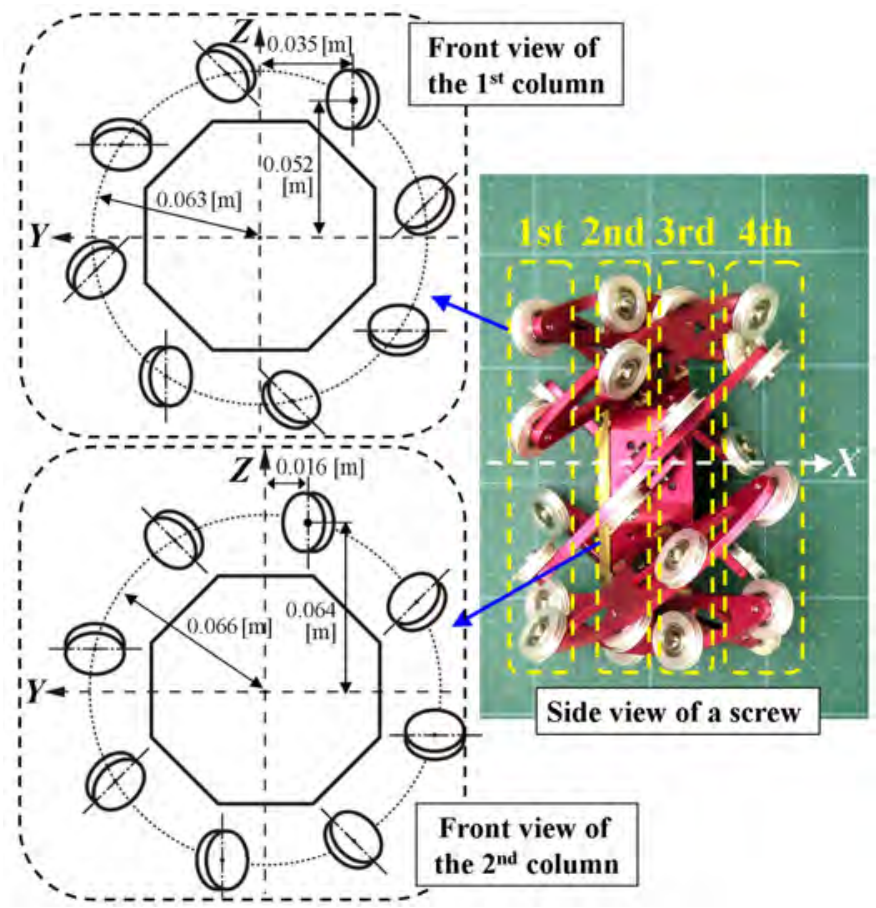

Fig. 7. Side and front views of a screw (left screw drive unit)
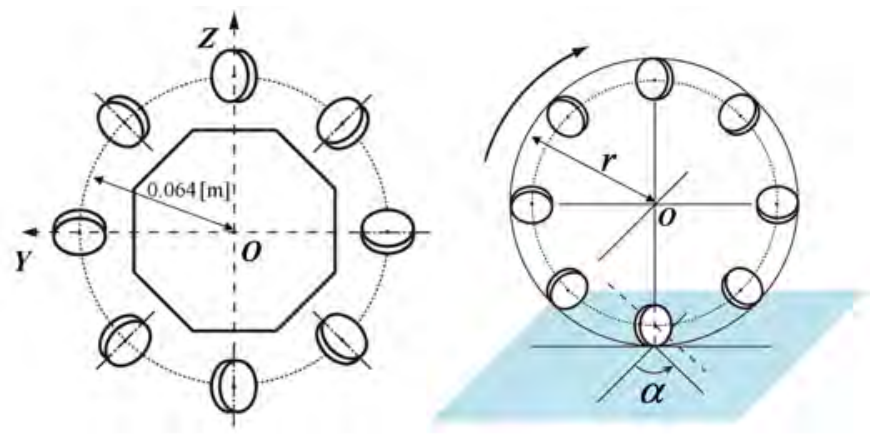

Fig. 8. Simplified screw model (left: front view, right: relation to the ground)

As shown in Fig. 6, we define the local coordinate system $O-X Y Z$ attached to a screw drive unit. The $X$ axis is set along the rotation axis of the screw, and the positive direction of the $X$ axis points towards the back of the screw. The $Y$ and $Z$ axes are set so as to pass through the centers of the sides of the octagonal prism. We also define $\alpha\left(-\frac{\pi}{2}<\alpha \leq \frac{\pi}{2}\right)$ as the angle of the blade from the $X$ axis when viewed from the outside of the screw, as shown on the left of Fig. 6. If a unit has positive (negative) $\alpha$, we refer to it as a left (right) screw drive unit. Further, $\alpha_{i}$ is defined as the angle $\alpha$ of the $i$ th screw unit.

As shown in the side view of the screw on the right of Fig. 7, a screw unit has four columns of passive wheels. In each column, the passive wheels are aligned on a circle in a plane perpendicular to the rotation axis of the screw. Two figures on the left of Fig. 7 show the front views of the wheels in the first and second columns. The center of each passive wheel is located on a circle shown in the dotted line. Each wheel is inclined at $\alpha$ about the axis shown in a dash-dotted 


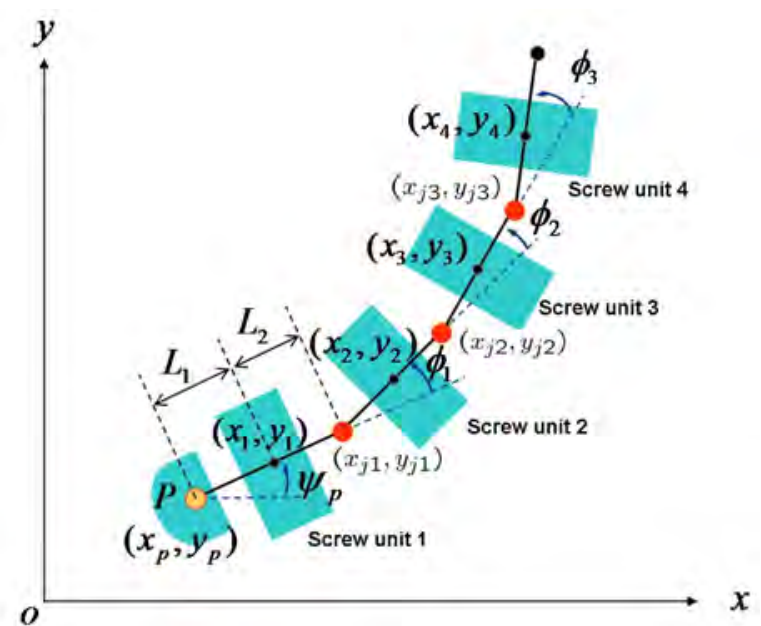

Fig. 9. Definition of coordinate variables

line, which passes through the center of the passive wheel and is perpendicular to a side of the octagonal prism. Note that the positions of the wheels in the third (fourth) column are symmetric to the ones in the second (first) column with respect to $Z$ axis. As a result, the wheels in the first (second) column are located at similar positions on the $Y-Z$ plane to the wheels in the third (fourth) column. It is also seen from Fig. 7 that the distance from the rotation axis of the screw to the passive wheels in the second and third columns are longer than the distance to the wheels in the first and fourth columns. This implies that if the ground is flat and horizontal, and if the pitch angles of the joints are controlled to 0 , a wheel in the second column and one in the third column alternately contact with the ground. Further, at the moments when the wheel contacting with the ground is switched, two wheels contact with the ground at the same time. However, it is difficult to construct a model taking into account such switching properties of the passive wheel in contact with the ground. Thus, in order to describe the average behavior of the screw unit, we assume that the passive wheels as shown on the left of Fig. 8 exist at $X=0$ in the middle of the second and the third columns, and that only one of these wheels contacts with the ground without side slip. Also, we assume that a perpendicular line from $O$ to the ground passes through the contact point with the ground, as shown on the right of Fig. 8. In this case, the rotation axis of the passive wheel on the ground is parallel to the ground, so that its projection to the ground is inclined at $\alpha$ from the rotation axis of the screw.

Due to the assumptions mentioned above on the relationship between the passive wheels and the ground, our model used in this paper has a limitation in describing the real robot, even in the case where the ground is flat and horizontal. Further, since the ground is not completely flat in reality, two or more of the passive wheels of one unit can contact with the ground. In such situations, it is difficult for the units to change the orientation without side slip of passive wheels. Despite these complex properties of the robot, we start with a simpler model for control design based on the assumptions mentioned above, since a complex model describing the robot more exactly is not easy to obtain and is not necessarily useful for control

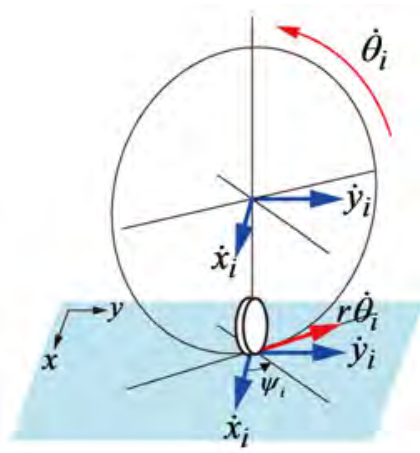

Fig. 10. Velocities generated for a passive wheel

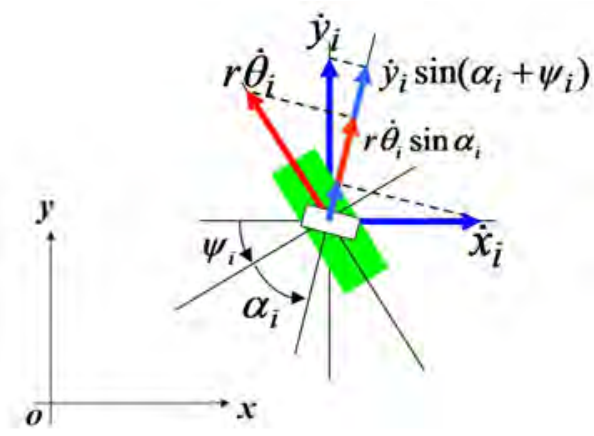

Fig. 11. Velocity constraint for a passive wheel (top view)

design.

\section{Kinematic Model}

In this section, we derive a kinematic model of the robot composed of 4 screw drive units described in Section II.

As shown in Fig. 9, let $o$ be the origin of the absolute coordinate system, $P$ be the point to be controlled in the head of the robot, $o-x y$ be the absolute coordinate system. Also, let $\left[\begin{array}{lll}x_{p} & y_{p} & \psi_{p}\end{array}\right]^{T}$ be the absolute coordinate of $P$ and the orientation of the unit 1 . The positions of the center of the screw unit $i$ and the joint $i$ are defined as $\left[\begin{array}{ll}x_{i} & y_{i}\end{array}\right]^{T}$ and $\left[\begin{array}{ll}x_{j i} & y_{j i}\end{array}\right]^{T}$, respectively. Furthermore, let $L_{1}$ be the length from the front tip of each link to the center of the screw drive unit on the link, and $L_{2}$ be the length from the center of the screw unit to the rear end of the link. The joint angle $\phi_{i}$ is defined as the orientation of the unit $i$ with respect to the unit $i-1$, and $\psi_{i}=\psi_{p}+\sum_{k=1}^{i-1} \phi_{k}(i=$ $2,3,4)$ denotes the orientation of the unit $i$ with respect to the absolute coordinate system. Additionally, let $\dot{\theta}_{i}(i=1,2,3,4)$ be the angular velocity of the screw drive unit $i$.

The position of the center of the screw unit $i$ is described from a geometrical relation as follows:

$$
\begin{aligned}
& x_{i}=x_{p}+L_{1} \cos \psi_{p}+\sum_{j=1}^{i-1}\left(L_{2} \cos \psi_{j}+L_{1} \cos \psi_{j+1}\right) \\
& y_{i}=y_{p}+L_{1} \sin \psi_{p}+\sum_{j=1}^{i-1}\left(L_{2} \sin \psi_{j}+L_{1} \sin \psi_{j+1}\right),
\end{aligned}
$$

where $\psi_{1}:=\psi_{p}$. Since it is assumed that the passive wheels do not slip sideways, we need to take into account the velocity 


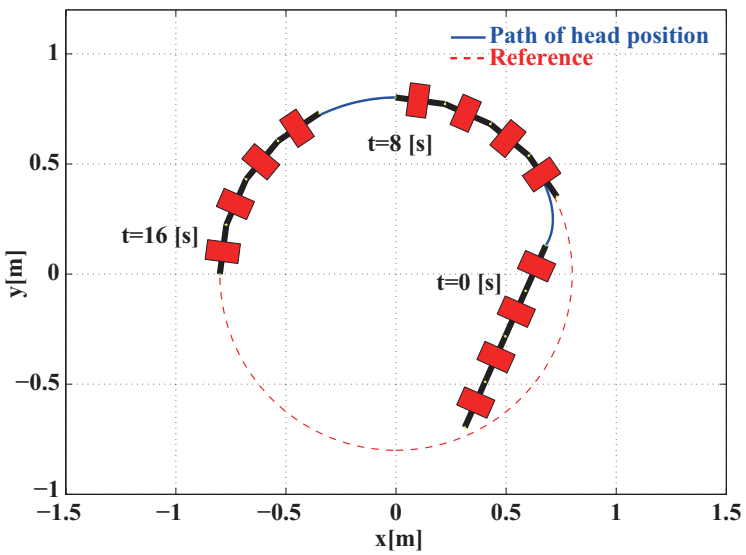

Fig. 12. $x-y$ plot of the head position in simulation (feedback)
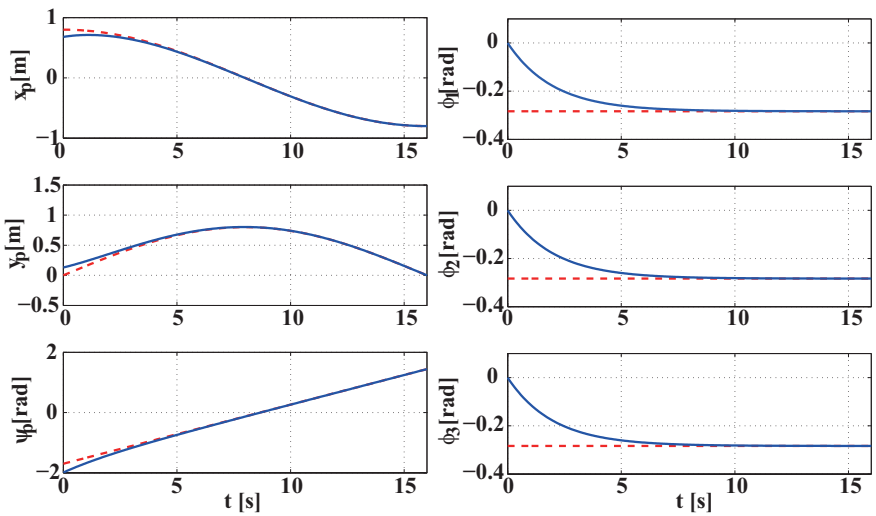

Fig. 13. Time responses of the state variables in simulation (feedback)

constraint condition. The velocity constraint condition here is more complicated than conventional snake-like robots [6]-[8] because of the screw units. As shown in Fig. 10, if the screw drive unit $i$ rotates at angular velocity $\dot{\theta}_{i}$, the velocity $r \dot{\theta}_{i}$ is generated for the passive wheel, where $r$ denotes the radius of the screw drive unit (distance from the rotation axis of the unit to the ground) as shown in Fig. 8. At the same time, if the center of the screw unit moves with the velocity $\left(\dot{x}_{i}, \dot{y}_{i}\right)$, the same velocity is generated for the passive wheel. Fig. 11 shows the top view of the passive wheel on the ground and the velocities generated for the passive wheel. The component of the velocity $r \dot{\theta}_{i}$ in the direction of the axle of the passive wheel is $r \dot{\theta}_{i} \sin \alpha_{i}$. The $x-$ and $y$-components $\left(\dot{x}_{i}, \dot{y}_{i}\right)$ of the translational velocity of the unit $i$ respectively generate $\dot{x}_{i} \cos \left(\alpha_{i}+\psi_{i}\right)$ and $\dot{y}_{i} \sin \left(\alpha_{i}+\psi_{i}\right)$ in the direction of the axle of the passive wheel. Therefore, the velocity constraint is described as follows:

$$
\dot{x}_{i} \cos \left(\alpha_{i}+\psi_{i}\right)+\dot{y}_{i} \sin \left(\alpha_{i}+\psi_{i}\right)+r \dot{\theta}_{i} \sin \alpha_{i}=0 .
$$

By substituting the derivatives of (1) into (2), the following kinematic model is obtained:

$$
A \dot{\xi}=B u,
$$

where $\xi=\left[\begin{array}{llllll}x_{p} & y_{p} & \psi_{p} & \phi_{1} & \phi_{2} & \phi_{3}\end{array}\right]^{T}$ is the state vector to be controlled, and $u=\left[\begin{array}{lllllll}\dot{\theta}_{1} & \dot{\theta}_{2} & \dot{\theta}_{3} & \dot{\theta}_{4} & \dot{\phi}_{1} & \dot{\phi}_{2} & \dot{\phi}_{3}\end{array}\right]^{T}$ is the control input vector. The system matrices $A$ and $B$ are defined as follows:

$$
\begin{aligned}
& A:=\left[\begin{array}{c|c}
A_{11} & A_{12} \\
\hline 0 & E_{3}
\end{array}\right], \quad B:=\operatorname{block} \operatorname{diag}\left(B_{1}, E_{3}\right) \\
& A_{11}:=\left[\begin{array}{lll}
a_{11} & a_{12} & a_{13} \\
a_{21} & a_{22} & a_{23} \\
a_{31} & a_{32} & a_{33} \\
a_{41} & a_{42} & a_{43}
\end{array}\right], A_{12}:=\left[\begin{array}{ccc}
0 & 0 & 0 \\
a_{24} & 0 & 0 \\
a_{34} & a_{35} & 0 \\
a_{44} & a_{45} & a_{46}
\end{array}\right] \\
& B_{1}:=-r \operatorname{diag}\left(\sin \alpha_{1}, \sin \alpha_{2}, \sin \alpha_{3}, \sin \alpha_{4}\right) \\
& a_{i 1}:=\cos \left(\alpha_{i}+\psi_{i}\right), \quad a_{i 2}:=\sin \left(\alpha_{i}+\psi_{i}\right) \\
& a_{13}:=L_{1} \sin \alpha_{1}, \quad a_{23}:=L \sin \left(\alpha_{2}+\phi_{1}\right)+L_{1} \sin \alpha_{2} \\
& a_{24}:=L_{1} \sin \alpha_{2}, \quad a_{33}:=L \sin \left(\alpha_{3}+\phi_{1}+\phi_{2}\right)+a_{34} \\
& a_{34}:=L \sin \left(\alpha_{3}+\phi_{2}\right)+L_{1} \sin \alpha_{3}, \quad a_{35}:=L_{1} \sin \alpha_{3} \\
& a_{43}:=L \sin \left(\alpha_{4}+\phi_{1}+\phi_{2}+\phi_{3}\right)+a_{44} \\
& a_{44}:=L \sin \left(\alpha_{4}+\phi_{2}+\phi_{3}\right)+a_{45} \\
& a_{45}:=L \sin \left(\alpha_{4}+\phi_{3}\right)+L_{1} \sin \alpha_{4}, \quad a_{46}:=L_{1} \sin \alpha_{4}
\end{aligned}
$$

where $L:=L_{1}+L_{2}$, and $E_{k}$ denotes the identity matrix of size $k$. In our experimental system, the values of parameters are $L_{1}=0.103[\mathrm{~m}], L_{2}=0.123[\mathrm{~m}], r=0.075[\mathrm{~m}], \alpha_{i}=-\frac{\pi}{4}$ $[\operatorname{rad}](i=1,3), \alpha_{i}=\frac{\pi}{4}[\operatorname{rad}](i=2,4)$.

Remark 1: If the upper part of the screw unit contacts with the environment in the same way as the lower part, we have another velocity constraint

$$
\dot{x}_{i} \cos \left(-\alpha_{i}+\psi_{i}\right)+\dot{y}_{i} \sin \left(-\alpha_{i}+\psi_{i}\right)-r \dot{\theta}_{i} \sin \left(-\alpha_{i}\right)=0
$$

for the passive wheel in contact with the ceiling. From this constraint together with (2), we obtain

$$
\begin{aligned}
& \dot{x}_{i} \cos \psi_{i}+\dot{y}_{i} \sin \psi_{i}+r \dot{\theta}_{i} \tan \alpha_{i}=0 \\
& \dot{x}_{i} \sin \psi_{i}-\dot{y}_{i} \cos \psi_{i}=0 .
\end{aligned}
$$

Note that the second equation implies that the component, which is perpendicular to the link, of the velocity $\left(\dot{x}_{i}, \dot{y}_{i}\right)$ at the center of the unit is 0 . This implies that the upper and lower parts cooperatively drive the unit into the direction along the rotation axis, in contrast to most crawler units whose upper and lower parts generate the velocity in the opposite directions at the center of the unit.

\section{TRAJECTORY CONTROL}

For the system in (3), a control law for trajectory tracking is designed as follows:

$$
u=B^{-1} A\left(\dot{\xi}_{d}-K e\right),
$$

where $e=\xi-\xi_{d}, \xi_{d}$ is a given target trajectory, and $K$ is a given feedback gain matrix. We notice that $B$ is invertible if $\alpha_{i} \neq 0(i=1,2,3,4)$.

By substituting (5) into (3), the closed-loop system is given as follows:

$$
A(\dot{e}+K e)=0 .
$$

If the matrix $A$ has full column rank, then it holds $\dot{e}+K e=0$. Therefore $\xi \rightarrow \xi_{d}(t \rightarrow \infty)$ is guaranteed if $K$ is positive definite. On the other hand, if $A$ is not of full column rank, the convergence of $\xi$ is not guaranteed, since $\dot{e}+K e=0$ does not necessarily hold. Appendix A describes a necessary condition 
of the joint angles $\left(\phi_{1}, \phi_{2}\right)$, for which $A$ does not have full column rank. As mentioned in the end of Appendix A, the necessary condition is satisfied in the case where the robot has a zig-zag shape with $\phi_{1}-\phi_{2}>2.35$ [rad] and $\phi_{2}<-1.35$ [rad], or in the case where $\phi_{i} \simeq \frac{\pi}{2}$ or $-\frac{\pi}{2}(i=1,2)$. In our target applications such as searches in narrow spaces, the target angles are typically chosen away from these values, since these values require wider space for the robot to pass through.

\section{A. Numerical Examples}

We show an example of our simulation results where the target path of the head position $P$ is given as an arc of radius $R_{p}=0.8[\mathrm{~m}]$, as shown in Fig.12. Target joint angles are chosen such that each joint position tracks the circular path if $P$ tracks it. More precisely, the target trajectory $\xi_{d}$ is chosen as

$$
\begin{aligned}
& \xi_{d}=\left[R_{p} \cos \frac{\pi}{16} t, R_{p} \sin \frac{\pi}{16} t, \frac{\pi}{16} t-\frac{\pi}{2}-\frac{\phi_{d}}{2}, \phi_{d}, \phi_{d}, \phi_{d}\right]^{T} \\
& \phi_{d}:=-2 \sin ^{-1} \frac{L}{2 R_{p}}=-0.283 .
\end{aligned}
$$

The feedback gain in (5) and the initial state are $K=0.5 E_{6}$ and $\xi(0)=[1.48,0.13,-1.99,0,0,0]^{T}$, respectively.

Fig.12 shows an $x-y$ plot of the trajectory of the head position $P$, and Fig. 13 indicates the time responses of the state variables. The solid and dashed lines in the figures show the state responses and the target trajectories, respectively. From these figures, it can be seen that the state variables converge to the desired trajectory, and the robot moves along the target path.

\section{B. Experiments}

We first compare the responses of the head position $P$ by feedforward and feedback control for fixed joint angles. The position and orientation of the head $\left[x_{p}, y_{p}, \psi_{p}\right]^{T}$ are measured by a vision sensor system (QuickMag IV, OKK). The target trajectory $\xi_{d}$ is chosen as

$$
\xi_{d}=\left[R_{p} \cos \frac{\pi}{12} t, R_{p} \sin \frac{\pi}{12} t, \frac{\pi}{12} t-\frac{\pi}{2}, 0,0,0\right]^{T}, \quad R_{p}=0.7 .
$$

Since both the initial and target angles of joints are 0 [rad], joint angles are fixed to $\phi_{1}=\phi_{2}=\phi_{3}=0$ [rad]. Fig. 14 shows an $x-y$ plot of the head position $P$ (left column) and the time responses of $\left(x_{p}, y_{p}, \psi_{p}\right)$ (right column). The solid line shows the response by feedback control for $K=0.5 E_{6}$, whereas dash-dotted line shows the response by feedforward control, i.e. $K=0$. In Fig. 14, the response by feedforward control is significantly slower than the target trajectory, which causes large tracking error of $P$. This shows that our model is not correct enough to describe the real system. If the model is correct, we have $\dot{e}=0$ from (6) for $A$ of full column rank. Since the initial tracking error is 0 in this example, $\xi$ should always be equal to $\xi^{d}$ even if $K=0$. A possible reason for this modeling error is that the assumptions for the passive wheels on the ground do not hold. On the other hand, all the variables are well controlled to the target trajectories by applying the feedback control. Thus, the uncertainty of our model can be considered to be within the allowable level for control design. Construction of more complex models describing the real
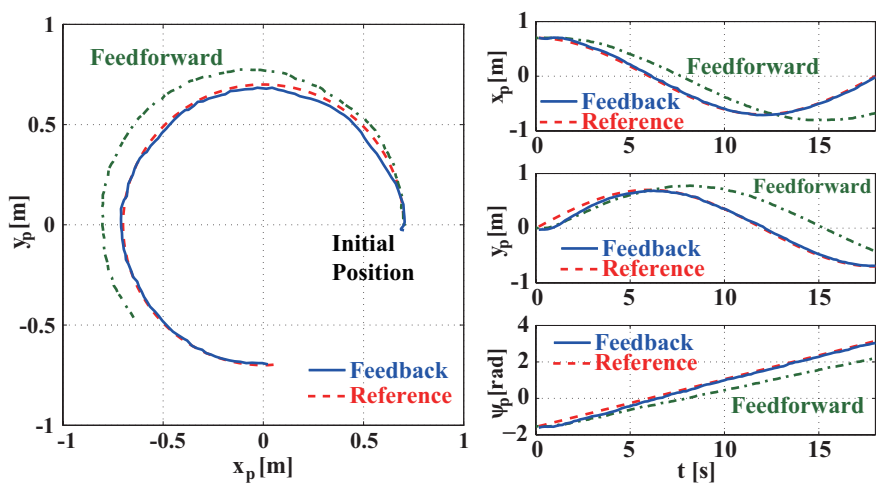

Fig. 14. Experimental results for fixed joint angles (feedback \& feedforward)

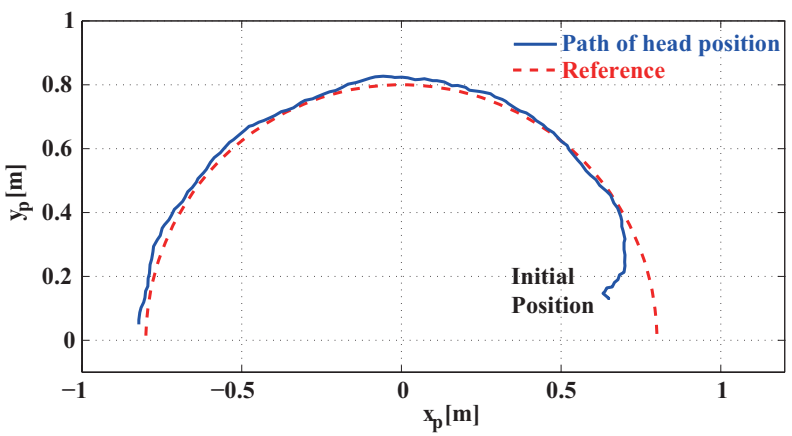

Fig. 15. $x-y$ plot of the head position in experiment (feedback)
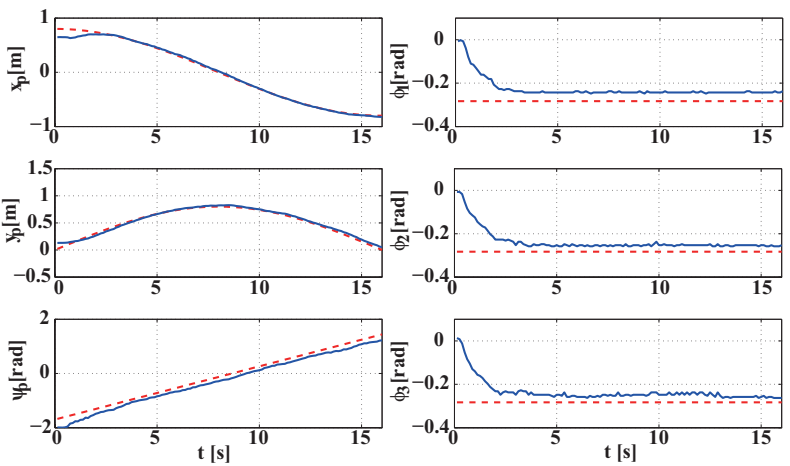

Fig. 16. Time responses of the state variables in experiment (feedback)

robot more exactly and control design based on such complex models are possible future works.

Next, we show a similar case to the numerical example in Section IV-A where the joint angles are changed. The same values of $\xi_{d}, \xi(0)$ and $K$ are chosen as in Section IV-A. Fig. 15 shows an $x-y$ plot of the head position $P$, and Fig. 16 indicates the time responses of the state variables. These figures show that the head position $P$ tracks the target trajectory with similar performance to the fixed joint case in Fig. 14. However, the steady-state error which is not seen in the simulation result in Fig. 13, is caused for each state. Possible reasons, except for violation of the assumptions on the passive wheels as mentioned above, are slight rotation of the body inside the screw units and the load due to the communications cables. 


\section{Front-Unit-Following CONTROL}

In Section IV, a feedback control system to steer the head position and orientation as well as joint angles to given target values has been designed based on a kinematic model. However, in typical practical situations where a human operator manipulates the robot watching images from a camera attached to the head, it is hard for operators to give commands for all joints as well as the head position and orientation, such that the shape of the robot is adapted to narrow spaces.

In this section, we propose a front-unit-following control method for the snake-like robot using the screw drive mechanism, and show numerical examples and experimental results to evaluate the effectiveness of the proposed method.

\section{A. Control Objective}

The main goal in this section is to fit the robot shape to the path of the front unit for given $\left(\dot{x}_{p}, \dot{y}_{p}, \dot{\psi}_{p}\right)$, by controlling the joint angles. In order to fit the robot shape to the path of the front unit, it is desired that each joint is controlled to the path of the head position $P$. However, since $\dot{\psi}_{p}$ is not a manipulate variable but given in advance, it is difficult to control joint 1 to the path $\gamma^{\prime}$ of $P$, as shown in Fig. 17. Thus, we aim to determine $\left(\dot{\phi}_{1}, \dot{\phi}_{2}, \dot{\phi}_{3}\right)$ such that each joint follows the path $\gamma$ of the joint 1 . Also, we determine $\left(\dot{\theta}_{1}, \dot{\theta}_{2}, \dot{\theta}_{3}, \dot{\theta}_{4}\right)$ which realizes the given $\left(\dot{x}_{p}, \dot{y}_{p}, \dot{\psi}_{p}\right)$.

In particular, we focus on two special cases as follows:

Case (i): A typical situation in manual operation where $\left(\dot{x}_{p}, \dot{y}_{p}, \dot{\psi}_{p}\right)$ are given as

$$
\dot{x}_{p}=-v_{1} \cos \psi_{p}, \quad \dot{y}_{p}=-v_{1} \sin \psi_{p}, \quad \dot{\psi}_{p}=\omega_{1},
$$

using the velocity commands $\left(v_{1}, \omega_{1}\right)$ given by a human operator. A control method without using measurement data of $\left(x_{p}, y_{p}, \psi_{p}\right)$ is typically required in this case, since a human operator often determines $\left(v_{1}, \omega_{1}\right)$ watching images from a camera attached to the robot, and no sensor for measuring $\left(x_{p}, y_{p}, \psi_{p}\right)$ is available.

Case (ii): If $\left(x_{p}, y_{p}, \psi_{p}\right)$ are measured, the following feedback law can be applied

$$
\dot{\xi}_{1}=\dot{\xi}_{d 1}-K_{1}\left(\xi_{1}-\xi_{d 1}\right), \quad \xi_{1}:=\left[x_{p}, y_{p}, \psi_{p}\right]^{T},
$$

where $K_{1}$ is a feedback gain and $\xi_{d 1}$ is a given target trajectory of $\xi_{1}$. This is a similar situation to Section IV, where a target trajectory of $\left(x_{p}, y_{p}, \psi_{p}\right)$ is given in advance.

\section{B. Decision of Control Input}

Assume that joint positions $\left(x_{j 2}, y_{j 2}\right),\left(x_{j 3}, y_{j 3}\right),\left(x_{j 4}, y_{j 4}\right)$ are initially on the path $\gamma$ of the first joint $\left(x_{j 1}, y_{j 1}\right)$ at $t=$ 0 , as shown in Fig. 17. Then, path tracking is accomplished at each time $t \geq 0$, if the velocity of each joint is always generated in tangential direction of $\gamma$.

From (7) and a geometric relationship

$$
\begin{aligned}
& x_{j 1}=x_{p}+L \cos \psi_{p} \\
& y_{j 1}=y_{p}+L \sin \psi_{p},
\end{aligned}
$$

the target velocity of the joint 1 is described as

$$
\begin{aligned}
& \dot{x}_{j 1}=\dot{x}_{p}-L \dot{\psi}_{p} \sin \psi_{p} \\
& \dot{y}_{j 1}=\dot{y}_{p}+L \dot{\psi}_{p} \cos \psi_{p} .
\end{aligned}
$$

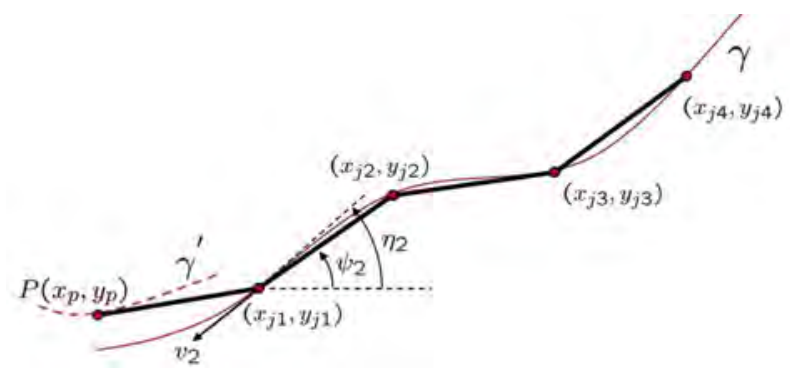

Fig. 17. Target path $\gamma$ and joint positions

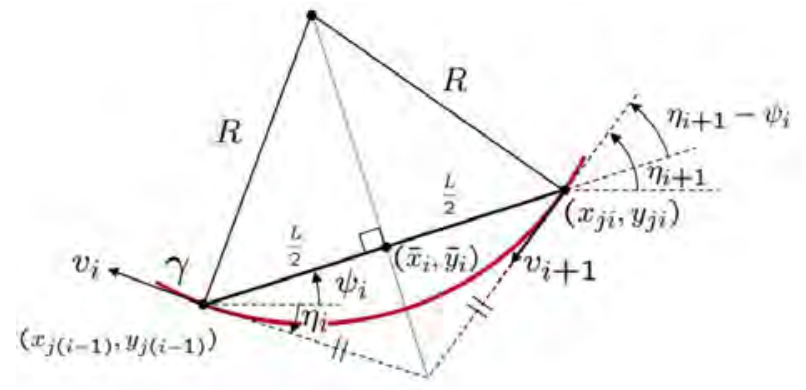

Fig. 18. Target path between two joints

Let $\eta_{i+1}$ denote the orientation of the tangent vector of $\gamma$ at the position $\left(x_{j i}, y_{j i}\right)$ of the joint $i(i=2,3)$. Then the target translational velocity $v_{i+1}$ of the joint $i$ and the target angular velocity $\dot{\psi}_{i}$ of the unit $i$ need to satisfy

$$
\begin{aligned}
& \dot{x}_{j i}=\dot{x}_{j(i-1)}-L \dot{\psi}_{i} \sin \psi_{i}=-v_{i+1} \cos \eta_{i+1} \\
& \dot{y}_{j i}=\dot{y}_{j(i-1)}+L \dot{\psi}_{i} \cos \psi_{i}=-v_{i+1} \sin \eta_{i+1} .
\end{aligned}
$$

By solving (11), $\dot{\psi}_{i}$ and $v_{i+1}$ for path-tracking are derived as

$$
\begin{aligned}
\dot{\psi}_{i} & =\frac{\dot{x}_{j(i-1)} \sin \eta_{i+1}-\dot{y}_{j(i-1)} \cos \eta_{i+1}}{L \cos \left(\psi_{i}-\eta_{i+1}\right)} \\
v_{i+1} & =-\frac{\dot{x}_{j(i-1)} \cos \psi_{i}+\dot{y}_{j(i-1)} \sin \psi_{i}}{\cos \left(\psi_{i}-\eta_{i+1}\right)} .
\end{aligned}
$$

Note that $\eta_{i+1}$ is equivalent to a past value of $\eta_{i}$, since the coordinate $\left(x_{j i}, y_{j i}\right)$ of the joint $i$ is a position where the joint $i-1$ passed in the past. Thus, in order to obtain $\eta_{i+1}$, the past data, e.g. $\left(\dot{x}_{j 1}, \dot{y}_{j 1}\right)$ in (10), needs to be stored.

In this paper, we adopt a simpler algorithm without using past data, by assuming that the transition rate of curvature of the target path $\gamma$ is sufficiently small between two consecutive joints. In such cases, it is satisfied that

$$
\eta_{i+1}-\psi_{i}=\psi_{i}-\eta_{i}
$$

since the directions of $v_{i+1}$ and $v_{i}$ are symmetric with respect to the link $i$, as shown in Fig. 18. Also, since (11) implies

$$
\begin{aligned}
& v_{i+1} \cos \left(\eta_{i+1}-\psi_{i}\right)=-\dot{x}_{j i} \cos \psi_{i}-\dot{y}_{j i} \sin \psi_{i} \\
& =-\dot{x}_{j(i-1)} \cos \psi_{i}-\dot{y}_{j(i-1)} \sin \psi_{i}=v_{i} \cos \left(\psi_{i}-\eta_{i}\right),
\end{aligned}
$$

we have $v_{i+1}=v_{i}$ from (14). Therefore, at the middle point $\left(\bar{x}_{i}, \bar{y}_{i}\right)$ of each link, the translational velocity $\bar{v}_{i}$ is generated along the link as follows

$$
\begin{aligned}
\dot{\bar{x}}_{j i} & =\dot{x}_{j(i-1)}-\frac{L}{2} \dot{\psi}_{i} \sin \psi_{i}=-\bar{v}_{i} \cos \psi_{i} \\
\dot{\bar{y}}_{j i} & =\dot{y}_{j(i-1)}+\frac{L}{2} \dot{\psi}_{i} \cos \psi_{i}=-\bar{v}_{i} \sin \psi_{i} .
\end{aligned}
$$


By solving (16), we have

$$
\begin{aligned}
& \dot{\psi}_{i}=\frac{2}{L}\left\{\dot{x}_{j(i-1)} \sin \psi_{i}-\dot{y}_{j(i-1)} \cos \psi_{i}\right\} \\
& \bar{v}_{i}=-\dot{x}_{j(i-1)} \cos \psi_{i}-\dot{y}_{j(i-1)} \sin \psi_{i} .
\end{aligned}
$$

Since $\dot{\phi}_{i}=\dot{\psi}_{i+1}-\dot{\psi}_{i}$, input variables $\left(\dot{\phi}_{1}, \dot{\phi}_{2}, \dot{\phi}_{3}\right)$ are recursively obtained for given $\left(\dot{x}_{p}, \dot{y}_{p}, \dot{\psi}_{p}\right)$. Once $\left(\dot{\phi}_{1}, \dot{\phi}_{2}, \dot{\phi}_{3}\right)$ are determined, the screws' angular velocities $\left(\dot{\theta}_{1}, \cdots, \dot{\theta}_{4}\right)$ in $u$ can be obtained from the first 4 rows in (3) as follows:

$$
\dot{\theta}=B_{1}^{-1} A_{1} \dot{\xi}
$$

where $\theta:=\left[\theta_{1}, \theta_{2}, \theta_{3}, \theta_{4}\right]^{T}$ and $A_{1}:=\left[A_{11}, A_{12}\right]$.

In Case (i), it holds from (7), (10) and (17) that

$$
\begin{aligned}
& \bar{v}_{2}=v_{1} \cos \phi_{1}-L \omega_{1} \sin \phi_{1} \\
& \dot{\psi}_{2}=-\frac{2}{L}\left(v_{1} \sin \phi_{1}+L \omega_{1} \cos \phi_{1}\right) .
\end{aligned}
$$

In the same way, velocity commands for the rest of units are recursively determined as

$$
\begin{aligned}
& \bar{v}_{i}=\bar{v}_{i-1} \cos \phi_{i-1}-\frac{L}{2} \dot{\psi}_{i-1} \sin \phi_{i-1} \\
& \dot{\psi}_{i}=-\frac{2}{L} \bar{v}_{i-1} \sin \phi_{i-1}-\dot{\psi}_{i-1} \cos \phi_{i-1}
\end{aligned}
$$

for $i=3,4$. Since $\dot{\psi}_{2}=\dot{\psi}_{p}+\dot{\phi}_{1}$, the target angular velocity of the joint 1 , which achieves $\dot{\psi}_{2}$ in (19), is written as

$$
\dot{\phi}_{1}=-\frac{2}{L} v_{1} \sin \phi_{1}-\omega_{1}\left(2 \cos \phi_{1}+1\right) .
$$

Also from $\dot{\psi}_{3}=\dot{\psi}_{p}+\dot{\phi}_{1}+\dot{\phi}_{2}$, we have

$$
\begin{aligned}
& \dot{\phi}_{2}=-\frac{2}{L} \bar{v}_{2} \sin \phi_{2}-\left(\omega_{1}+\dot{\phi}_{1}\right)\left(\cos \phi_{2}+1\right) \\
& \bar{v}_{3}=\bar{v}_{2} \cos \phi_{2}-\frac{L}{2}\left(\omega_{1}+\dot{\phi}_{1}\right) \sin \phi_{2}
\end{aligned}
$$

using (20). In the same way, $\dot{\phi}_{3}$ is obtained as

$$
\dot{\phi}_{3}=-\frac{2}{L} \bar{v}_{3} \sin \phi_{3}-\left(\omega_{1}+\dot{\phi}_{1}+\dot{\phi}_{2}\right)\left(\cos \phi_{3}+1\right)
$$

using (20) and $\dot{\psi}_{4}=\dot{\psi}_{p}+\dot{\phi}_{1}+\dot{\phi}_{2}+\dot{\phi}_{3}$. From (21)-(23), it can be seen that $\left(\dot{\phi}_{1}, \dot{\phi}_{2}, \dot{\phi}_{3}\right)$ can be determined without measurement of $\left(x_{p}, y_{p}, \psi_{p}\right)$. Also, although $A_{1}$ and $\dot{\xi}$ depend on $\psi_{p}$, it is canceled in (18), since it holds from (4) and (7) that

$$
a_{i 1} \dot{x}_{p}+a_{i 2} \dot{y}_{p}=v_{1} \cos \left(\alpha_{i}+\psi_{i}-\psi_{p}\right), \quad i=1,2,3,4 .
$$

Thus, measurement of $\left(x_{p}, y_{p}, \psi_{p}\right)$ is not necessary to determine $\dot{\theta}$ in the case where $\left(\dot{x}_{p}, \dot{y}_{p}, \dot{\psi}_{p}\right)$ are given as in (7).

In Case (ii), the closed-loop system for $\xi_{1}$ is obtained from (8) and (18) as

$$
A_{11}\left(\dot{e}_{1}+K_{1} e_{1}\right)=0, \quad e_{1}:=\xi_{1}-\xi_{d 1} .
$$

Thus, if $A_{11}$ has full column rank, $\xi_{1}$ converges to $\xi_{d 1}$ as $t$ increases.

\section{Convergence for Constant Curvature}

The target velocities in Section V-B are derived under assumptions that joint positions are initially on the target path $\gamma$, and that the transition rate of curvature of $\gamma$ is sufficiently small between two consecutive joints. This implies that a rapid change of curvature causes a large path tracking error. Therefore, it is important to find conditions where off-tracking can be recovered by the proposed control law.
In this section, we show that even if joint positions are initially off the target path $\gamma$, they converge to $\gamma$ in the case where the curvature of $\gamma$ is constant. More precisely, we consider the case where $\left(v_{1}, \omega_{1}\right)$ is constant in (7). We assume that the robot moves forward, i.e. $v_{1}>0$. The extension of the method to other cases is a subject of future research. Also, we note that only the case of $\omega_{1}<0$ is described, since the case of $\omega_{1}>0$ is similar. The case of $\omega_{1}=0$, where the path is a straight line, is also omitted, since it is easier to be proved.

By integrating (7), the head position $P$ is obtained as

$$
x_{p}=C_{x}+R_{p} \sin \psi_{p}, \quad y_{p}=C_{y}-R_{p} \cos \psi_{p}
$$

where $R_{p}:=-\frac{v_{1}}{\omega_{1}}>0$ and

$$
C_{x}:=x_{0}-R_{p} \sin \psi_{0}, \quad C_{y}:=y_{0}+R_{p} \cos \psi_{0} .
$$

In (27), $\left(x_{0}, y_{0}, \psi_{0}\right)$ denotes the initial value of $\left(x_{p}, y_{p}, \psi_{p}\right)$. Thus, the position of the joint 1 is obtained from (9) as

$$
\begin{aligned}
& x_{j 1}=C_{x}+R_{j 1} \sin \left(\psi_{p}+\alpha_{j 1}\right) \\
& y_{j 1}=C_{y}-R_{j 1} \cos \left(\psi_{p}+\alpha_{j 1}\right),
\end{aligned}
$$

where $R_{j 1}=\sqrt{R_{p}^{2}+L^{2}}$ and $\alpha_{j 1}$ is a value satisfying

$$
\cos \alpha_{j 1}=\frac{R_{p}}{R_{j 1}}, \quad \sin \alpha_{j 1}=\frac{L}{R_{j 1}} .
$$

Thus from $L>0, R_{p}>0$, we assume $0<\alpha_{j 1}<\frac{\pi}{2}$ without loss of generality. It is seen from (28) that the joint 1 moves along a circle of radius $R_{j 1}$ and center $\left(C_{x}, C_{y}\right)$. Also, it holds from (21) that

$$
\dot{\phi}_{1}=\frac{2 R_{j 1} \omega_{1}}{L} \sin \left(\phi_{1}-\alpha_{j 1}\right)-\omega_{1} .
$$

Now define $V_{1}:=\frac{1}{2} \Phi_{1}^{2}$ for $\Phi_{1}:=\dot{\phi}_{1}$, which satisfies $V_{1}>0$ for $\Phi_{1} \neq 0$. Then, it holds

$$
\dot{V}_{1}=\Phi_{1} \dot{\Phi}_{1}=\Phi_{1}^{2} \frac{2 R_{j 1} \omega_{1}}{L} \cos \left(\phi_{1}-\alpha_{j 1}\right) .
$$

Since $-\frac{\pi}{2} \leq \phi_{1} \leq \frac{\pi}{2}$ due to the movable range of joints, we consider two cases where $-\frac{\pi}{2} \leq \phi_{1} \leq 0$ and $0 \leq \phi_{1} \leq \frac{\pi}{2}$. In the case of $-\frac{\pi}{2} \leq \phi_{1} \leq 0$, we have $-\pi<\phi_{1}-\alpha_{j 1}<0$ since $0<\alpha_{j 1}<\frac{\pi}{2}$. This implies $\sin \left(\phi_{1}-\alpha_{j 1}\right)<0$. Thus, the first term on the right hand side of (30) is positive, since $\omega_{1}<0$. Therefore, in the case of $-\frac{\pi}{2} \leq \phi_{1} \leq 0$, it always holds from (30) that $\dot{\phi}_{1}>-\omega_{1}>0$, so that $\phi_{1}$ asymptotically becomes nonnegative. Therefore, it is sufficient to consider the case of $0 \leq \phi_{1} \leq \frac{\pi}{2}$. In this case, it holds $\cos \left(\phi_{1}-\alpha_{j 1}\right)>0$, since $-\frac{\pi}{2}<\phi_{1}-\alpha_{j 1}<\frac{\pi}{2}$. Thus, we have $\dot{V}_{1}<0\left(\forall \Phi_{1} \neq 0\right)$, and this implies $\Phi_{1}=\dot{\phi}_{1} \rightarrow 0$ from Lyapunov's stability theorem. Therefore, it is seen from (30) that

$$
\phi_{1} \rightarrow \sin ^{-1} \frac{L}{2 R_{j 1}}+\alpha_{j 1}(t \rightarrow \infty) .
$$

In the same way, it can be derived for $\phi_{i}(i=2,3)$ that

$$
\phi_{2} \rightarrow 2 \sin ^{-1} \frac{L}{2 R_{j 1}}, \quad \phi_{3} \rightarrow 2 \sin ^{-1} \frac{L}{2 R_{j 1}} .
$$

See Appendix B for the detail on the convergence of $\phi_{2}$ and $\phi_{3}$.

The asymptotic value of $\phi_{i}$, which results from (32)-(33), is illustrated in Fig. 19. Since the orientation $\psi_{p}$ of the unit 


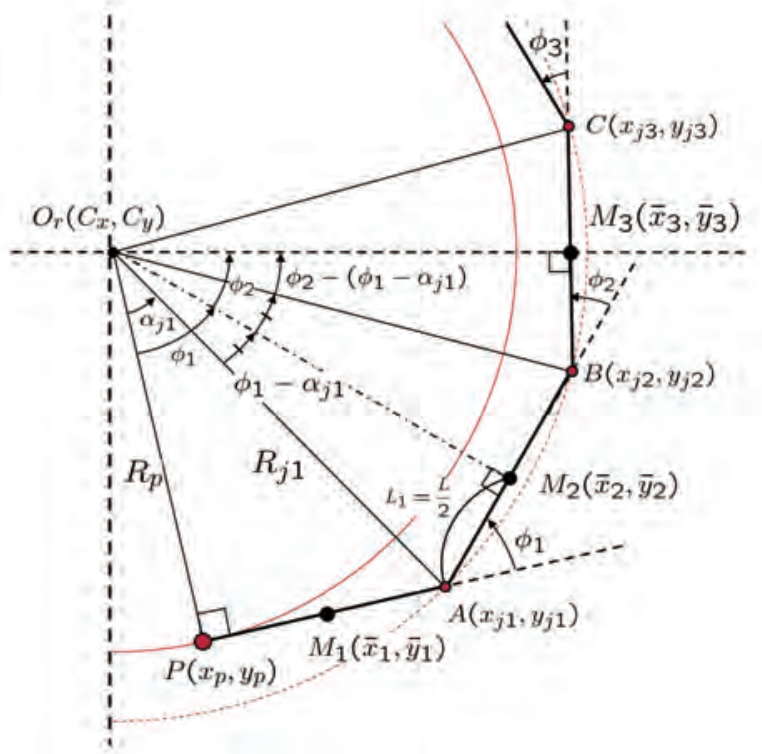

Fig. 19. Configuration asymptotically achieved for constant curvature

1 is always equivalent to the tangent direction of the path of $P$, it holds $\angle O_{r} P A=\frac{\pi}{2}$. This implies from (29) that $\angle O_{r} A P=\frac{\pi}{2}-\alpha_{j 1}$. Therefore, $\angle O_{r} A B=\frac{\pi}{2}-\phi_{1}+\alpha_{j 1}$. Let $M_{2}$ denote the foot of perpendicular from $O_{r}$ to $\overline{A B}$, then $\angle A O_{r} M_{2}=\phi_{1}-\alpha_{j 1}$. Now, since we have $\overline{A M_{2}}=$ $R_{j 1} \sin \left(\phi_{1}-\alpha_{j 1}\right)=L / 2$ from (32), $M_{2}$ is also the midpoint of $\overline{A B}$ as well as the foot of the perpendicular, which implies $\triangle O_{r} A M_{2} \equiv \triangle O_{r} B M_{2}$. Thus, since $\triangle O_{r} A B$ is an isosceles triangle with $\overline{O_{r} A}=\overline{O_{r} B}$, the first and second joints are on the same circular path of radius $R_{j 1}$. Also, it holds from $\angle O_{r} A B=\angle O_{r} B A=\pi / 2-\phi_{1}+\alpha_{j 1}$ that $\angle O_{r} B C=$ $\pi / 2-\phi_{2}+\phi_{1}-\alpha_{j 1}$, which implies $\angle B O_{r} M_{3}=\phi_{2}-\phi_{1}+\alpha_{j 1}$ for the foot of perpendicular $M_{3}$ from $O_{r}$ to $\overline{B C}$. From (32)(33), it holds

$$
\begin{aligned}
& \overline{B M}_{3}=R_{j 1} \sin \left(\phi_{2}-\left(\phi_{1}-\alpha_{j 1}\right)\right) \\
& =R_{j 1} \sin \left(2 \sin ^{-1}\left(\frac{L}{2 R_{j 1}}\right)-\sin ^{-1}\left(\frac{L}{2 R_{j 1}}\right)\right)=\frac{L}{2}
\end{aligned}
$$

which implies $M_{3}$ is the midpoint of $\overline{B C}$. Thus, the joint 3 is on the same circle as the joint 1 and 2 , since $\triangle O_{r} B C$ is an isosceles triangle with $\overline{O_{r} B}=\overline{O_{r} C}$. Furthermore, it is trivial to show the joint 4 at the tail is also on the same circle, since $\phi_{2}=\phi_{3}$ from (33).

Note that it is difficult to guarantee the convergence to the target paths except for circular paths. However, the control law is expected to work well, if the curvature of the path does not changes rapidly and if there is no initial tracking error. This is because the angular velocity of each joint angle is determined under the assumptions that (i) all the joints are currently on the target path and (ii) the transition rate of curvature of the target path is sufficiently small between two consecutive joints, as mentioned in Section V-B. Sections V-D and V-E show examples in which the control method works well for noncircular paths.

It is also important to note that the difficulty of guaranteeing the convergence of tracking error arises because the target path is not available in our control problem. If the target

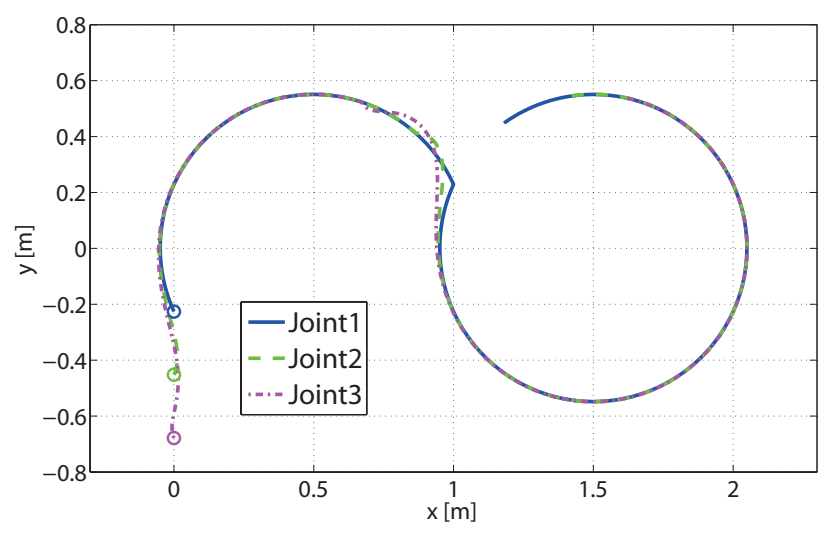

Fig. 20. $x-y$ plot of each joint path in simulation (feedforward)

path is available, it should be easier to derive a control law using the target path information such that the convergence is guaranteed for more general target paths. However, since a human operator typically gives velocity commands in real time to the front unit in our target applications, the target path needs to be estimated based on the memory of the past commands, which is difficult in many cases due to the computational burden. Thus, the proposed control method is not based on the past commands but only the current velocity command.

\section{Numerical Examples}

The proposed method is tested for two types of target paths. The first example adopts the connected arcs as the target path. The arcs are chosen to verify the convergence of tracking error, which is theoretically shown in Section V-C. On the other hand, the second example adopts the target path whose curvature continuously changes, rather than a circular target path. Although the convergence of the tracking error is not theoretically guaranteed for such target paths, the control law is expected to work well, if the curvature of the path does not changes rapidly and if there is no initial tracking error, as mentioned in the end of Section V-C. The target path in the second example is adopted to show the effectiveness of the control method for the target paths except for circular arcs. It is also important to note that information on the target paths is not used for control in both simulations in this section and experiments in the next section. Only the velocity commands $\left(v_{1}, \omega_{1}\right)$ at the current time and joint angles $\phi_{i}(i=1,2,3)$ are used in simulations for applying the proposed control law. The measured values of $\left(x_{p}, y_{p}, \psi_{p}\right)$ at the current time are additionally used for experiments in the next section.

The command for translational velocity $v_{1}$ of the front unit is given as $v_{1}=\frac{\pi}{60}[\mathrm{~m} / \mathrm{s}]$. The angular velocity is given as $\omega_{1}=-\frac{\pi}{30}[\mathrm{rad} / \mathrm{s}]$ for $t<30[\mathrm{~s}]$, then it is switched to $\omega_{1}=\frac{\pi}{30}[\mathrm{rad} / \mathrm{s}]$ for $t \geq 30$ [s]. This implies that the path of $P$ is composed of two connected arcs of radius 0.5 [m]. As mentioned in Section V-C, the target path $\gamma$ which is the path of joint 1 is an arc of radius $\sqrt{R_{p}^{2}+L^{2}}$, when the path of $P$ is an arc of radius $R_{p}$. Fig. 20 shows the paths of the joints for $0 \leq t \leq 85$ [s], where the initial state is $\xi(0)=\left[0,0, \frac{3}{2} \pi, 0,0,0\right]^{T}$. The solid, dashed and dash-dotted 


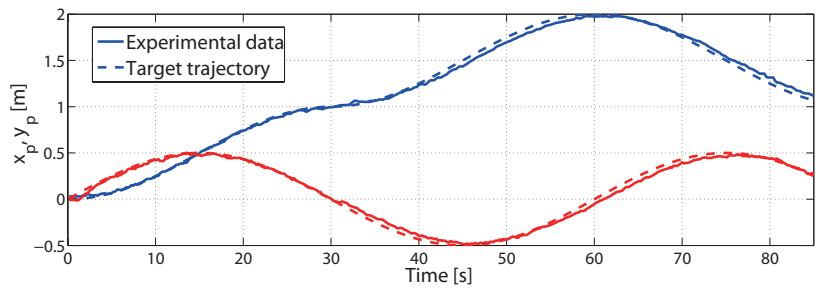

Fig. 21. Time plot of $x_{p}$ and $y_{p}$ in experiment (feedback)

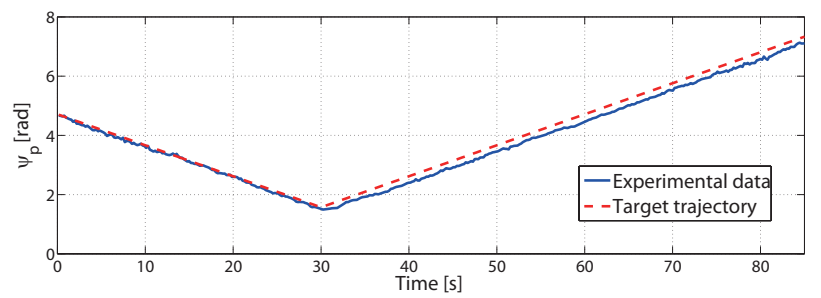

Fig. 22. Time plot of $\psi_{p}$ in experiment (feedback)

lines show the paths of the joint 1,2 and 3, respectively, and "o" denotes the initial position of each joint. Although the joint 2 and 3 initially deviate from the path of joint 1 due to the straight-line configuration, the tracking errors converge to 0 before $t=30$ [s] when $\omega_{1}$ is switched. Also, although the joint 2 and 3 are off the target path at $t=30$ [s] due to the jump of the curvature of the target path around $(1,0.25)$, the tracking errors converge to 0 without feedback control, once the curvature of the target path becomes constant, as mentioned in Section V-C.

Next, we show an example where the curvature of the target path is continuously changing. The command for translational velocity $v_{1}$ for the front unit is given as $v_{1}=\frac{\pi}{60}[\mathrm{~m} / \mathrm{s}]$, while the angular velocity is $\omega_{1}=-\frac{\pi}{30} \cos \lambda \frac{\pi}{60} t[\mathrm{rad} / \mathrm{s}]$ for a constant $\lambda$. This implies that $\omega_{1}$ is changed from $-\frac{\pi}{30}[\mathrm{rad} / \mathrm{s}]$ to $\frac{\pi}{30}[\mathrm{rad} / \mathrm{s}]$ in $60 / \lambda[\mathrm{s}]$. Table I shows the maximum tracking error for $\lambda=0.5,1,1.5,2$. It can be seen from Table I that e.g. for $\lambda \leq 1$, path tracking error is within $5.80 \times 10^{-3}[\mathrm{~m}]$, i.e. $4 \%$ of the width of screw drive units $2 r=0.15$ [m]. Also, we learn from this table that the maximum error tends to increase linearly with $\lambda$. Note that although we have simply used cosine functions to change the curvature of target path, many other types of paths, which human operators possibly give in practical situations, need to be considered in the future.

TABLE I

TRACKING ERROR FOR $\omega_{1}=-\frac{\pi}{30} \cos \lambda \frac{\pi}{60} t$

\begin{tabular}{|c|c|c|c|c|}
\hline & $\lambda=0.5$ & $\lambda=1$ & $\lambda=1.5$ & $\lambda=2$ \\
\hline$\left(x_{j 2}, y_{j 2}\right)$ & $1.22 \times 10^{-3}$ & $1.98 \times 10^{-3}$ & $2.99 \times 10^{-3}$ & $4.07 \times 10^{-3}$ \\
$\left(x_{j 3}, y_{j 3}\right)$ & $2.35 \times 10^{-3}$ & $3.92 \times 10^{-3}$ & $5.94 \times 10^{-3}$ & $8.07 \times 10^{-3}$ \\
$\left(x_{j 4}, y_{j 4}\right)$ & $3.34 \times 10^{-3}$ & $5.80 \times 10^{-3}$ & $8.81 \times 10^{-3}$ & $1.20 \times 10^{-2}$ \\
\hline
\end{tabular}

\section{E. Experiments}

In order to compare with the simulation results in Section $\mathrm{V}-\mathrm{D}$, the same velocity commands generated in advance are given for the front unit, although our experimental system is

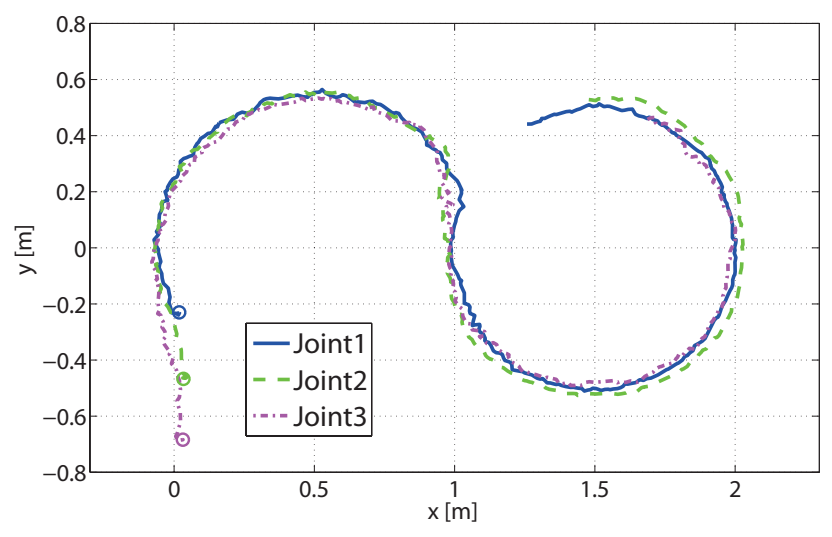

Fig. 23. $x-y$ plot of each joint path in experiment (feedback)

equipped with a joystick for a human operator to give velocity commands. A major difference from the numerical example is that due to the effects of modeling errors and disturbances, the feedback law as in (8) is necessary to generate a similar target path $\gamma$ to the one in Section V-D. More precisely, the commands for the front unit $\left(\dot{x}_{p}, \dot{y}_{p}, \dot{\psi}_{p}\right)$ are given as in (8) using measurement data $\left(x_{p}, y_{p}, \psi_{p}\right)$ by a vision sensor system (QuickMag IV, OKK), where

$$
\dot{x}_{p}^{d}=-v_{1} \cos \psi_{p}, \quad \dot{y}_{p}^{d}=-v_{1} \sin \psi_{p}, \quad \dot{\psi}_{p}^{d}=\omega_{1},
$$

and the feedback gain is chosen as $K_{1}=\operatorname{diag}(1,1,0.5)$. Similarly to Section V-D, the command for translational velocity $v_{1}$ for the front unit is given as $v_{1}=\frac{\pi}{60}[\mathrm{~m} / \mathrm{s}]$. The angular velocity is given as $\omega_{1}=-\frac{\pi}{30}[\mathrm{rad} / \mathrm{s}]$ for $t<30$ [s], then it is switched to $\omega_{1}=\frac{\pi}{30}[\mathrm{rad} / \mathrm{s}]$ for $t \geq 30$ [s]. Fig. 21 and Fig. 22 show the time responses of the head position and orientation, where the solid and dashed lines indicate the measurements $\left(x_{p}, y_{p}, \psi_{p}\right)$ and the target trajectories $\left(x_{p}^{d}, y_{p}^{d}, \psi_{p}^{d}\right)$, respectively. The paths of the joints for $0 \leq t \leq 85$ [s] in this case are shown in Fig. 23. The solid, dashed and dash-dotted lines show the paths of the joint 1,2 and 3, respectively, and "o" denotes the initial position of each joint. It can be seen that the similar responses to the ones in Fig. 20 are obtained in Fig. 23. However, in contrast to the simulation result where there is no steady-state error, the maximum steady-state error is nearly $5[\mathrm{~cm}]$ for the left turn. A possible reason for this is the steady-state error of $\left(x_{p}, y_{p}, \psi_{p}\right)$, which is slightly larger for the left turn at $t \geq 30$, as shown in Fig. 21 and Fig. 22. The front-unit-following controller in this paper does not take into account such steady-state error due to modeling error and disturbance. As a result, the radius of arc which the joint 1 tracks is approximately 5 [cm] less than the target arc.

In the next example, the command for translational velocity $v_{1}$ for the front unit is given as $v_{1}=\frac{\pi}{60}[\mathrm{~m} / \mathrm{s}]$, while the angular velocity is $\omega_{1}=-\frac{\pi}{30} \cos \lambda \frac{\pi}{60} t[\mathrm{rad} / \mathrm{s}]$ for $\lambda=1$. Fig. 24 shows paths of the joints for $0 \leq t \leq 95$ [s], where the initial state is $\xi(0)=\left[0,0, \frac{5}{3} \pi, 0,0,0\right]^{T}$. In contrast that the maximum tracking error is less than $4[\mathrm{~mm}]$ in the simulation result as shown in Table. I, the maximum error is about $5[\mathrm{~cm}]$ in the experiment, similarly to the previous example in Fig. 


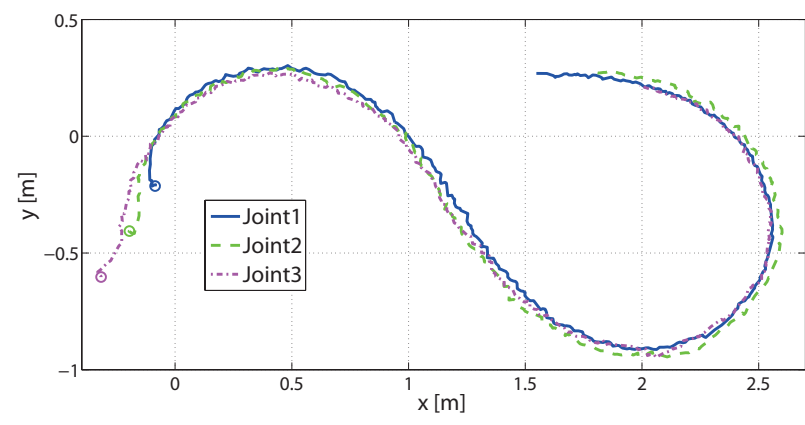

Fig. 24. $x-y$ plot for $\omega_{1}=-\frac{\pi}{30} \cos \frac{\pi}{60} t$ in experiment (feedback)

23. Developing a control method to handle modeling errors is one of the future issues.

\section{CONCLUSIONS}

In this paper, we have developed a new type of snake-like robot using screw-drive units connected by active joints. Also, a kinematic model has been derived and applied to trajectory tracking control. Furthermore, we have proposed a front-unitfollowing controller for steering each unit to follow the path of the front unit. This controller can support manual operations, since human operators are required to command only one unit in the front. Asymptotic convergence of the tracking error of the front-unit-following controller has been analyzed based on a Lyapunov approach for the case of constant curvature. The effectiveness of the control method has been demonstrated by numerical examples and experiments. Although the kinematic model and the controllers have been presented for 4 link robots in accordance with the experimental system, it is possible to extend these results to more general cases of $n(\geq 3)$ links. One of the future issues is to develop a control method to handle modeling errors due to the effects such as side slipping of the passive wheels. Also, the tracking performance of the front-unit-following controller needs to be investigated for many types of paths, which human operators possibly give in practical situations. Furthermore, this paper has only focused on the cases where the robot does not contact with environment except for the ground, which is assumed to be flat and horizontal. The experiments have been performed only on a flat floor of indoor environments, as the first step. A lot of problems including the improvement of the prototype need to be tackled in order that this robot is applicable outside of laboratory environments.

\section{ACKNOWLEDGEMENTS}

This research was supported in part by the Ministry of Education, Culture, Sports, Science and Technology Grantin-Aid for Scientific Research (No. 23360105). The authors thank H. Igarashi and M. Hara for assistance to set up the experimental system in this paper.

\section{APPENDIX}

\section{A. Necessary condition that $A$ is not of full column rank}

Since this paper considers the cases where $\alpha_{i}=-\frac{\pi}{4}$ $[\operatorname{rad}](i=1,3)$ and $\alpha_{i}=\frac{\pi}{4}[\operatorname{rad}](i=2,4)$, we can obtain the following fact.

Proposition 1: The matrix $A$ in (3) is not of full column rank, only if $\phi_{i}\left(-\frac{\pi}{2} \leq \phi_{i} \leq \frac{\pi}{2}, i=1,2\right)$ satisfies

$$
\phi_{2}=\sin ^{-1} \frac{D_{2}}{\Psi}-\beta_{2}
$$

where

$$
\begin{aligned}
& \Psi:=\sqrt{\left(L_{1} \cos \phi_{1}\right)^{2}+\left(L_{1} \sin \phi_{1}+L_{2}\right)^{2}} \\
& D_{2}:=\frac{L}{2}\left(\sin 2 \phi_{1}-\cos 2 \phi_{1}-1\right)-L_{1} \cos \phi_{1}
\end{aligned}
$$

and $\beta_{2}$ is the joint angles satisfying

$$
\sin \beta_{2}=\frac{L_{1} \sin \phi_{1}+L_{2}}{\Psi}, \quad \cos \beta_{2}=\frac{L_{1} \cos \phi_{1}}{\Psi} .
$$

Proof: From the definition of $A$, it is sufficient to discuss the column rank of $A_{11}$. Define $i$ th column of $A_{11}$ as $a_{i}$, i.e. $A_{11}=\left[a_{1}, a_{2}, a_{3}\right]$. Then, $a_{1}, a_{2}, a_{3}$ are linearly dependent if and only if there exist scalars $c_{1}, c_{2}, c_{3}$, which are not all zero and satisfy

$$
c_{1} a_{1}+c_{2} a_{2}+c_{3} a_{3}=0 .
$$

From the definition of $a_{1}$ and $a_{2}$, we have

$$
\begin{gathered}
a_{1}=a_{1}^{\prime} \cos \psi_{p}-a_{2}^{\prime} \sin \psi_{p}, \quad a_{2}=a_{1}^{\prime} \sin \psi_{p}+a_{2}^{\prime} \cos \psi_{p}, \\
a_{1}^{\prime}:=\left[\begin{array}{c}
\cos \alpha_{1} \\
\cos \left(\alpha_{2}+\phi_{1}\right) \\
\cos \left(\alpha_{3}+\phi_{1}+\phi_{2}\right) \\
\cos \left(\alpha_{4}+\phi_{1}+\phi_{2}+\phi_{3}\right)
\end{array}\right], \quad a_{2}^{\prime}:=\left[\begin{array}{c}
\sin \alpha_{1} \\
\sin \left(\alpha_{2}+\phi_{1}\right) \\
\sin \left(\alpha_{3}+\phi_{1}+\phi_{2}\right) \\
\sin \left(\alpha_{4}+\phi_{1}+\phi_{2}+\phi_{3}\right)
\end{array}\right] .
\end{gathered}
$$

By substituting (40) to (39), we have

$$
\begin{aligned}
& c_{1}^{\prime} a_{1}^{\prime}+c_{2}^{\prime} a_{2}^{\prime}+c_{3} a_{3}=0 \\
& c_{1}^{\prime}:=c_{1} \cos \psi_{p}+c_{2} \sin \psi_{p}, \quad c_{2}^{\prime}:=-c_{1} \sin \psi_{p}+c_{2} \cos \psi_{p} .
\end{aligned}
$$

This implies

$$
\left[\begin{array}{l}
c_{1} \\
c_{2}
\end{array}\right]=R\left(\psi_{p}\right)\left[\begin{array}{l}
c_{1}^{\prime} \\
c_{2}^{\prime}
\end{array}\right], \quad R\left(\psi_{p}\right):=\left[\begin{array}{cc}
\cos \psi_{p} & -\sin \psi_{p} \\
\sin \psi_{p} & \cos \psi_{p}
\end{array}\right],
$$

where $R\left(\psi_{p}\right)$ is nonsingular for any $\psi_{p}$. Thus, $c_{1}, c_{2}, c_{3}$ are not all zero and satisfy (39), if and only if $c_{1}^{\prime}, c_{2}^{\prime}, c_{3}$ are not all zero and satisfy (41). Therefore, $A_{11}$ does not have full column rank, only if $\phi_{1}$ and $\phi_{2}$ satisfy the equations in the first three raws of (41).

First, we consider the case of $c_{3}=0$. In this case, we can assume $c_{1}^{\prime}=1$ without loss of generality. By substituting $c_{1}^{\prime}=1$ and $\alpha_{1}=-\frac{\pi}{4}$ into the first row of (41), we obtain $c_{2}^{\prime}=-\frac{\cos \alpha_{1}}{\sin \alpha_{1}}=1$. Thus, from the second and the third rows of (41), we have

$$
\begin{aligned}
& \cos \left(\alpha_{2}+\phi_{1}\right)+\sin \left(\alpha_{2}+\phi_{1}\right)=0 \\
& \cos \left(\alpha_{3}+\phi_{1}+\phi_{2}\right)+\sin \left(\alpha_{3}+\phi_{1}+\phi_{2}\right)=0,
\end{aligned}
$$

respectively. By substituting $\alpha_{2}=\frac{\pi}{4}$ and $\alpha_{3}=-\frac{\pi}{4}$ into (42), we have $\cos \phi_{2}=0$ and $\sin \left(\phi_{1}+\phi_{2}\right)=0$. This implies $\phi_{i}=\frac{\pi}{2}$ or $-\frac{\pi}{2}(i=1,2)$, which satisfies $(36)$.

Next, we consider the case of $c_{3} \neq 0$. In this case, we can assume $c_{3}=1$ without loss of generality. It follows from $\alpha_{1}=-\frac{\pi}{4}$ and the first row of (41) that $c_{1}^{\prime}=c_{2}^{\prime}+L_{1}$. Thus, from the second and third rows of (41), we obtain

$$
\begin{aligned}
\left(c_{2}^{\prime}+L_{1}\right) \cos \left(\alpha_{2}+\phi_{1}\right)+\left(c_{2}^{\prime}+L\right) \sin \left(\alpha_{2}+\phi_{1}\right)+L_{1} \sin \alpha_{2}=0 \\
\left(c_{2}^{\prime}+L_{1}\right) \cos \left(\alpha_{3}+\phi_{1}+\phi_{2}\right)+\left(c_{2}^{\prime}+L\right) \sin \left(\alpha_{3}+\phi_{1}+\phi_{2}\right) \\
+L_{1} \sin \alpha_{3}+L \sin \left(\alpha_{3}+\phi_{1}\right)=0 .
\end{aligned}
$$



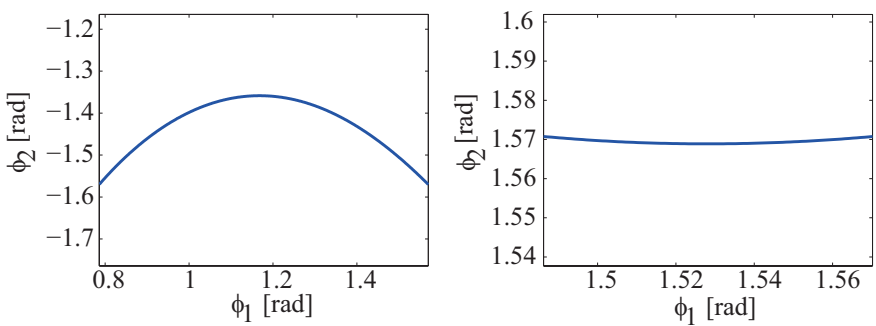

Fig. 25. $\left(\phi_{1}, \phi_{2}\right)$ for which $A$ does not have full column rank. (Left: $\phi_{1}>0$, $\phi_{2}<0$, Right: $\phi_{1}>0, \phi_{2}>0$ )

This implies from $\alpha_{2}=\frac{\pi}{4}$ and $\alpha_{3}=-\frac{\pi}{4}$ that

$$
\begin{aligned}
\left(2 c_{2}^{\prime}+L_{1}+L\right) \cos \phi_{1}+ & \left(L-L_{1}\right) \sin \phi_{1}+L_{1}=0 \\
\left(L_{1}-L\right) \cos \left(\phi_{1}+\phi_{2}\right)+ & \left(2 c_{2}^{\prime}+L_{1}+L\right) \sin \left(\phi_{1}+\phi_{2}\right) \\
& -L_{1}-L \cos \phi_{1}+L \sin \phi_{1}=0 .
\end{aligned}
$$

By substituting

$$
2 c_{2}^{\prime}+L_{1}+L=-\frac{L_{1}+\left(L-L_{1}\right) \sin \phi_{1}}{\cos \phi_{1}},
$$

which is obtained from (43), into (44), we have

$$
\begin{array}{r}
\left(L_{1}-L\right) \cos \phi_{2}-L_{1} \sin \left(\phi_{1}+\phi_{2}\right)-L_{1} \cos \phi_{1} \\
-L \cos ^{2} \phi_{1}+L \sin \phi_{1} \cos \phi_{1}=0 .
\end{array}
$$

Since (46) is written as an affine equation of $\sin \phi_{2}$ and $\cos \phi_{2}$ :

$$
\begin{array}{r}
L_{1} \cos \phi_{1} \sin \phi_{2}+\left(L_{1} \sin \phi_{1}+L-L_{1}\right) \cos \phi_{2} \\
=\frac{L}{2}\left(\sin 2 \phi_{1}-\cos 2 \phi_{1}-1\right)-L_{1} \cos \phi_{1},
\end{array}
$$

$\phi_{2}$ can be described as (36).

As shown in the proof, $A$ is not of full column rank for $\phi_{i}=\frac{\pi}{2}$ or $-\frac{\pi}{2}(i=1,2)$. Fig. 25 shows other sets of $\left(\phi_{1}\right.$, $\phi_{2}$ ) which satisfy (36). The figure on the left shows the case where $\phi_{1}>0$ and $\phi_{2}<0$, while the case, where $\phi_{1}>0$ and $\phi_{2}>0$, is shown on the right. Note that there is no set of $\left(\phi_{1}\right.$, $\phi_{2}$ ), which satisfies (36), in the case of $-\frac{\pi}{2}<\phi_{1}, \phi_{2}<0$ and the case of $-\frac{\pi}{2}<\phi_{1}<0$ and $0<\phi_{1}<\frac{\pi}{2}$.

As shown on the left of Fig. 25, in the case of $\phi_{1}>0$ and $\phi_{2}<0$, the robot has a zig-zag shape with $\phi_{1}-\phi_{2}>2.35$ [rad] and $\phi_{2}<-1.35$ [rad]. The figure on the right shows that (36) is satisfied only for $\phi_{1} \simeq \phi_{2} \simeq \frac{\pi}{2}$ in the case where $\phi_{1}>0$ and $\phi_{2}>0$.

\section{B. Convergence of Joint Angles $\phi_{2}$ and $\phi_{3}$}

As shown in Section $\mathrm{V}-\mathrm{C}$, the angle of the joint 1 converges to a constant value in (32). Therefore, we investigate the convergence properties of $\phi_{2}$ and $\phi_{3}$ for

$$
\phi_{1}=\sin ^{-1} \frac{L}{2 R_{j 1}}+\alpha_{j 1}, \quad \dot{\phi}_{1}=0 .
$$

From (17) and (48), it holds that

$$
\begin{aligned}
& \Phi_{2}:=\dot{\phi}_{2}=\dot{\psi}_{3}-\dot{\psi}_{p}-\dot{\phi}_{1} \\
& =\frac{2}{L}\left\{\dot{x}_{j 2} \sin \psi_{3}-\dot{y}_{j 2} \cos \psi_{3}\right\}-\omega_{1} .
\end{aligned}
$$

Since (11) and the derivative of (28) imply that

$$
\begin{aligned}
& \dot{x}_{j 2} \sin \psi_{3}-\dot{y}_{j 2} \cos \psi_{3}=\dot{x}_{j 1} \sin \psi_{3}-\dot{y}_{j 1} \cos \psi_{3} \\
& \quad-L \dot{\psi}_{2}\left(\sin \psi_{2} \sin \psi_{3}+\cos \psi_{2} \cos \psi_{3}\right) \\
& =R_{j 1} \omega_{1} \sin \left(\psi_{3}-\left(\psi_{p}+\alpha_{j 1}\right)\right)-L \omega_{1} \cos \phi_{2},
\end{aligned}
$$

we have

$$
\Phi_{2}=\frac{2 \omega_{1}}{L}\left\{R_{j 1} \sin \left(\phi_{1}+\phi_{2}-\alpha_{j 1}\right)-L \cos \phi_{2}\right\}-\omega_{1} .
$$

Furthermore, by substituting $\phi_{1}$ in (48) into (50), it holds

$$
\begin{aligned}
& \Phi_{2}=\frac{2 \omega_{1}}{L}\left\{R_{j 1} \sin \left(\sin ^{-1} \frac{L}{2 R_{j 1}}+\phi_{2}\right)-L \cos \phi_{2}\right\}-\omega_{1} \\
& =\frac{2 \omega_{1}}{L}\left\{R_{j 1} \cos \left(\sin ^{-1} \frac{L}{2 R_{j 1}}\right) \sin \phi_{2}-\frac{L}{2} \cos \phi_{2}\right\}-\omega_{1} \\
& =\frac{2 \omega_{1}}{L}\left\{\sqrt{R_{j 1}^{2}-\frac{L^{2}}{4}} \sin \phi_{2}-\frac{L}{2} \cos \phi_{2}\right\}-\omega_{1} \\
& =\frac{2 \omega_{1}}{L} R_{j 1} \sin \left(\phi_{2}+\alpha_{j 2}\right)-\omega_{1},
\end{aligned}
$$

where

$$
\alpha_{j 2}=\cos ^{-1} \frac{1}{R_{j 1}} \sqrt{R_{j 1}^{2}-\left(\frac{L}{2}\right)^{2}}=-\sin ^{-1} \frac{L}{2 R_{j 1}} .
$$

Thus from $R_{j 1}>L>0$, we assume $-\frac{\pi}{2}<\alpha_{j 2}<0$ without loss of generality. Then for $V_{2}:=\frac{1}{2} \Phi_{2}^{2}$, it holds

$$
\dot{V}_{2}=\Phi_{2} \dot{\Phi}_{2}=\Phi_{2}^{2} \frac{2 R_{j 1} \omega_{1}}{L} \cos \left(\phi_{2}+\alpha_{j 2}\right) .
$$

Using a similar procedure to the one for (31), it holds

$$
\Phi_{2}=\frac{2 \omega_{1}}{L} R_{j 1} \sin \left(\phi_{2}+\alpha_{j 2}\right)-\omega_{1} \rightarrow 0,
$$

since we have a constraint as $-\frac{\pi}{2} \leq \phi_{2} \leq \frac{\pi}{2}$. Therefore it holds from (54) that

$$
\phi_{2} \rightarrow \sin ^{-1} \frac{L}{2 R_{j 1}}-\alpha_{j 2}=2 \sin ^{-1} \frac{L}{2 R_{j 1}} .
$$

Now we investigate the convergence property of $\phi_{3}$ for $\phi_{2}=$ $2 \sin ^{-1} \frac{L}{2 R_{j 1}}$ and $\dot{\phi}_{2}=0$. Similarly to (49)-(51), it holds that

$$
\begin{aligned}
& \Phi_{3}:=\dot{\phi}_{3}=\dot{\psi}_{4}-\dot{\psi}_{p}-\dot{\phi}_{1}-\dot{\phi}_{2} \\
& =\frac{2 \omega_{1}}{L} R_{j 1} \sin \left(\phi_{3}+\alpha_{j 2}\right)-\omega_{1} .
\end{aligned}
$$

Since (51) and (56) have the same form, it holds that $\phi_{3} \rightarrow$ $2 \sin ^{-1} \frac{L}{2 R_{j 1}}$, similarly to (55).

\section{REFERENCES}

[1] T. Kamegawa, T. Yamasaki, H. Igarashi, and F. Matsuno, "Development of the snake-like rescue robot KOHGA," in Proc. IEEE Int. Conf. Robot. Autom., pp. 5081-5086, 2004.

[2] T. Takayama and S. Hirose, "Development of "Souryu I \& II" -connected crawler vehicle for inspection of narrow and winding space-," J. Robot. Mech., vol. 15, no. 1, pp. 61-69, 2001.

[3] K. Suzumori, S. Wakimoto and M.Takata, "A miniature inspection robot negotiating pipes of widely varying diameter" IEEE Int. Conf. Robot. Autom., pp. 2736-2740, 2003.

[4] A. Kuwada, S. Wakimoto, K. Suzumori, and Y. Adomi, "Automatic pipe negotiation control for snake-like robot," in Proc. IEEE/ASME Int. Conf. Advanced Intelligent Mechatronics, pp. 558-563, 2008.

[5] S. Hirose, Biologically inspired robots: snake-like locomotors and manipulators. Oxford, U.K.: Oxford Univ. Press, 1993.

[6] J. Ostrowski and J. Burdick, "Gait kinematics for a serpentine robot," in Proc. IEEE Int. Conf. Robot. Autom., pp. 1294-1299, 1996.

[7] P. Prautsch and T. Mita, "Control and analysis of the gait of snake robots," in Proc. IEEE Int. Conf. Control Appl., pp. 502-507, 1999.

[8] F. Matsuno and K. Mogi, "Redundancy controllable system and control of snake robots based on kinematic model," in Proc. IEEE Conf. Decision Control, pp. 4791-4796, 2000.

[9] S. Ma, "Analysis of creeping locomotion of a snake-like robot," $A d v$. Robot., vol. 15, no. 2, pp. 205-224, 2001.

[10] M. Saito, M. Fukaya, and T. Iwasaki, "Serpentine locomotion with robotic snakes," IEEE Control Syst. Mag., vol. 22, no. 1, pp. 64-81, 2002.

[11] T. Kamegawa and F. Matsuno, "Proposition of twisting mode of locomotion and GA based motion planning for transition of locomotion modes of 3-dimensional snake-like robot," in Proc. IEEE Int. Conf. Robot. Autom., pp. 1507-1512, 2002. 
[12] A. A. Transeth, R. I. Leine, C. Glocker, K. Y. Pettersen, and P. Liljebäck, "Snake robot obstacle-aided locomotion: modeling, simulations, and experiments," IEEE Trans. Robot., vol. 24, no. 1, pp. 88-104, 2008.

[13] R. L. Hatton and H. Choset, "Generating gaits for snake robots: annealed chain fitting and keyframe wave extraction" Auton. Robot., vol. 28, pp. 271-281, 2010.

[14] X. Wu and S. Ma, "Adaptive creeping locomotion of a CPG-controlled snake-like robot to environment change" Âuton. Robot., vol. 28, pp. 283 294, 2010.

[15] P. Liljebäck, K. Y. Pettersen, Ø. Stavdahl, and J. T. Gravdahl, "Controllability and stability analysis of planar snake robot locomotion," IEEE Trans. Automat. Contr., vol. 56, no. 6, pp. 1365-1380, 2011.

[16] K. Osuka and H. Kitajima, "Development of mobile inspection robot for rescue activities: MOIRA," in Proc. IEEE/RSJ Int. Conf. Intell. Robots Syst., pp. 3373-3377, 2003.

[17] J. Borenstein, M. Hansen, and A. Borrell, "The OmniTread OT-4 serpentine robot - design and performance," J. Field Robot., vol. 24, no. 7, pp. 601-621, 2007.

[18] S. Hirose, H. Ohno, T. Mitsui, and K. Suyama, "Design and experiments of in-pipe inspection vehicles for $\phi 25, \phi 50, \phi 250$ pipes," J. Robot. Mech., vol. 12, no. 3, pp. 310-317, 2000.

[19] T. Yamaguchi, Y. Kagawa, I. Hayashi, N. Iwatsuki, K. Morikawa, and K. Nakamura, "Screw principle microrobot passing steps in a small pipe," in Proc. Int. Symp. on Micromechatronics and Human Science, pp. 149-152, 1999.

[20] A. Kakogawa and S. Ma, "Mobility of an in-pipe robot with screw drive mechanism inside curved pipes," in Proc. IEEE Int. Conf. on Robotics and Biomimetics, pp. 1530-1535, 2010.

[21] F. Matsuno, and T. Kawai, "Propulsion system based on screw drive mechanism," Japanese published patent application 2003-293656, 2003.

[22] M. Sampei, T. Tamura, T. Kobayashi, and N. Shibui, "Arbitrary path tracking control of articulated vehicles using nonlinear control theory," IEEE Trans. Cont. Sys. Tech., vol.3, pp.125-131, 1995.

[23] C. Altafini, "A Path-tracking criterion for an LHD articulated vehicle," Int. J. Robot. Res., vol.18, pp.435-441, 1999.

[24] C. Altafini, "Path following with reduced off-tracking for multibody wheeled vehicles," IEEE Trans. Cont. Sys. Tech., vol.11, pp.598-605, 2003.

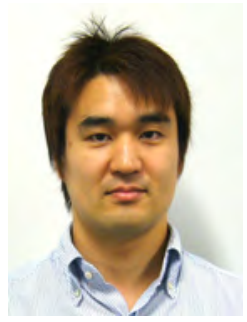

Hiroaki Fukushima received the B.S. and M.S. degrees in engineering and Ph.D. degree in informatics from Kyoto University, Japan, in 1995, 1998 and 2001, respectively. From 1999 to 2004 he was a Research Fellow of Japan Society for the Promotion of Science. From 2001 to 2003 he was a Visiting Scholar at University of California at San Diego. From 2004 to 2009 he worked as a Research Associate and Assistant Professor at the University of Electro-Communications, Japan. Currently he is an Assistant Professor of Kyoto University, Japan. His research interests include system identification and robust control. He received the ISCIE Young Author Award in 2001 and the SICE paper award in 2003. He is a member of the IEEE, SICE, ISCIE and RSJ.

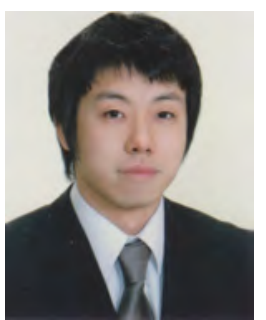

Shogo Satomura received the B.S. and M.S. degrees in engineering from University of ElectroCommunications, Japan, in 2004 and 2006, respectively. He is currently working at Canon Inc., Japan. His master's degree focused on rescue robot systems and snake-like robots.

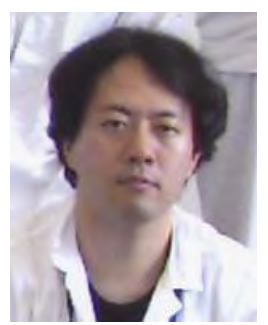

Toru Kawai received the B.S. degree in engineering from Tokyo University of Agriculture and Technology, Japan, in 2001, and M.S. degree in engineering from Tokyo Institute of Technology, Japan, in 2003. From 2003 to 2006, he was with Mitsubishi Heavy Industries, Ltd. Japan. He is currently working at Honda R\&D, Co. Ltd., Japan. His master's degree focused on rescue robot systems and snake-like robots.

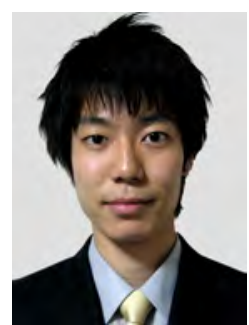

Motoyasu Tanaka received the B.S., M.S. and $\mathrm{Ph} . \mathrm{D}$. degrees in engineering from University of Electro-Communications, Japan, in 2005, 2007 and 2009, respectively. $\mathrm{He}$ is currently working at Canon Inc., Japan. His research interests include robotics and control, especially snake-like robots. He received Hatakeyama Memorial Prize and Miura Memorial Prize from The Japan Society of Mechanical Engineers in 2004 and 2006, and the IEEE Robotics and Automation Society Japan Chapter Young Award from IEEE RAS Japan Chapter in 2006. He is a member of the RSJ.-

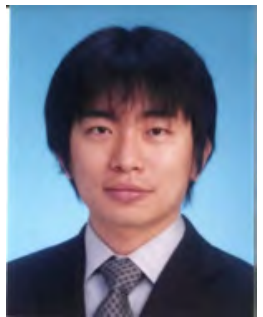

Tetsushi Kamegawa received the B.S., M.S. and $\mathrm{Ph} . \mathrm{D}$. degree in engineering from Tokyo Institute of Technology, Japan, in 1999, 2001 and 2004, respectively. In 2004 he was a Visiting Scholar of Roma University, Italy. From 2004 to 2006 he was a Researcher at International Rescue System Institute, Japan. From 2006 to 2009 he worked as a Research Associate, Assistant Professor and Senior Assistant Professor at the Okayama University, Japan. His research interests include snake robot and rescue robot. He received the SICE Interactive Organized Session Paper Awards in 2008. He is a member of the JSME, RSJ, SICE, and ISCIE.

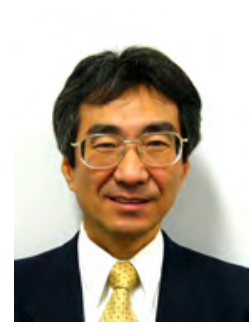

Fumitoshi Matsuno received the $\mathrm{PhD}$ (Dr. Eng.) degree from Osaka University in 1986. In 1986 he joined the Department of Control Engineering, Osaka University. He became a Lecturer in 1991 and an Associate Professor in 1992, in the Department of Systems Engineering, Kobe University. In 1996 he joined the Department of Computational Intelligence and Systems Science, Interdisciplinary Graduate School of Science and Engineering, Tokyo Institute of Technology as an Associate Professor. In 2003 he became a Professor in the Department of Mechanical Engineering and Intelligent Systems, University of ElectroCommunications. Since 2009, he has been a Professor in the Department of Mechanical Engineering and Science, Kyoto University. He holds also a post of the Vice-President of NPO International Rescue System Institute (IRS). His current research interests lie in robotics, control of distributed parameter system and nonlinear system, rescue support system in fire and disaster, and geographic information system. Dr. Matsuno received many awards including the Outstanding Paper Award in 2001 and 2006, Takeda Memorial Prize in 2001 from the Society of Instrument and Control Engineers. He is a member of the IEEE, the SICE, the JSME, the RSJ, the ISCIE, among other organizations. $\mathrm{He}$ is a co-chair of IEEE RAS Technical Committee on Safety, Security, and Rescue Robotics, a chair of Steering Committee of SICE Annual Conference, an editor of Journal of Intelligent and Robotic Systems, an associate editor of Advanced Robotics, International Journal of Control, Automation, and Systems, etc. and on the Conf. Editorial Board of IEEE CSS 